\title{
REGULARITY PROPERTIES OF JUMP DIFFUSIONS WITH IRREGULAR COEFFICIENTS.
}

\author{
GUOHUAN ZHAO
}

\begin{abstract}
In this paper, we investigate the regularity properties of strong solutions to SDEs driven by Lévy processes with irregular drift coefficients. Under some mild conditions, we show that the singular SDE has a unique strong solution for each starting point and the family of all the solutions forms a stochastic flow. Moreover, the Malliavin differentiability of the strong solutions is also obtained. As an application, we also prove a path-by-path uniqueness result for some related random ODEs. Our proofs are based on Schauder's estimate for the corresponding resolvent equation, which is also analytically interesting in itself.
\end{abstract}

Keywords: Stochastic flow, Lévy process, Zvonkin's transformation, Malliavin differentiability

AMS 2010 Mathematics Subject Classification: 60H10, 35R09

\section{INTRODUCTION}

1.1. Motivation and main results. The motivation of this paper is to study the following stochastic differential equation (SDE) driven by Lévy process with irregular drift coefficient

$$
\mathrm{d} X_{t}(x)=b\left(X_{t}(x)\right) \mathrm{d} t+\sigma\left(X_{t-}(x)\right) \mathrm{d} Z_{t}, X_{0}(x)=x \in \mathbb{R}^{d} .
$$

Here $Z$ is a $d$-dimensional pure jump Lévy process with Lévy-Khintchine triplet $(0,0, v), b: \mathbb{R}^{d} \rightarrow \mathbb{R}^{d}$ and $\sigma: \mathbb{R}^{d} \rightarrow \mathbb{R}^{d} \otimes \mathbb{R}^{d}$ are measurable maps. Two basic questions about SDEs with jumps as in (1.1) are its (strong) well-posedness and the regularity properties of the solutions $X_{t}(x, \omega)$ with respect to (w.r.t.) the initial value $x$ and the sample point $\omega$. When the coefficients are regular, the reader is referred to Kunita's fundamental work [Kun04] for the existence and regularity of stochastic flows associated to SDEs driven by general Poisson random measures. This paper mainly focuses on the case when the drift coefficients are irregular. In the case that $Z_{t}$ is a truncated rotational symmetric $\alpha$-stable process with $\alpha \in(1,2), \sigma=\mathbb{I}$ and $b \in C_{b}^{\beta}$ with $\beta>2-\alpha$, Haadem and Proske [HP14] proved the existence and Malliavin differentiability of $X_{t}(x, \omega)$. Another aim of this paper is to extend Haadem and Proske's results to more general (truncated) $\alpha$-stable like process $Z$ and relax the restrictions on $\alpha, \beta$ at the same time.

To achieve our goals, in this paper we first use a Zvonkin's change of variables to remove the irregular drift terms and transform the old SDEs to some new SDEs with regular coefficients, then

Research of Guohuan is supported by the German Research Foundation (DFG) through the Collaborative Research Centre(CRC) 1283 Taming uncertainty and profiting from randomness and low regularity in analysis, stochastics and their applications. 
we prove the desired properties of the solutions to (1.1) by studying the corresponding properties of the solutions to the new equation. From the probabilistic point of view, this approach can not be considered novel and the main idea can be traced back at least to [Zvo74]. However, in order to get the so-called Zvonkin's transform, we need to resort to some nice apriori estimates for the following resolvent equation associated to (1.1)

$$
\lambda u-\mathscr{L}^{b} u=f,
$$

where $\mathscr{L}^{b}$ is a Lévy type operator defined by

$$
\begin{aligned}
\mathscr{L}^{b} u(x) & :=\mathscr{L} u(x)+b(x) \cdot \nabla u(x) \\
& :=\int_{\mathbb{R}^{d}}\left[u(x+\sigma(x) z)-u(x)-\nabla u(x) \cdot \sigma(x) z \mathbf{1}_{B_{1}}(z)\right] v(\mathrm{~d} z)+b(x) \cdot \nabla u(x),
\end{aligned}
$$

and $v$ is the Lévy measure of the $\alpha$-stable like process $Z$. Noting that when $\alpha \in(0,1)$, the gradient $\nabla$ is of higher order than the nonlocal dissipative operator $\mathscr{L}$, the regularity theory for (1.2) is a highly nontrivial analytic problem when $\alpha \in(0,1)$.

To state our results precisely, we start by introducing the assumptions needed in this paper. Let $\alpha \in(0,2)$ and $Z$ be a Lévy process with Lévy-Khintchine triplet $(0,0, v)$.

$\left(\mathrm{A}_{1}\right) v$ is symmetric and there are some constants $c_{0}, \rho \in(0,1)$ such that

$$
\int_{B_{r}}\langle\theta, z\rangle^{2} v(\mathrm{~d} z) \geqslant c_{0} r^{2-\alpha}, \quad \forall r \in(0, \rho), \theta \in\left\{\vartheta \in \mathbb{R}^{d}:|\vartheta|=1\right\},
$$

and

$$
\int_{B_{r}}|z|^{2} v(\mathrm{~d} z) \leqslant c_{0}^{-1} r^{2-\alpha}, \quad \forall r \in(0, \rho),
$$

( $\left.\mathrm{A}_{2}\right) \sigma$ is non-degenerate and there is a constant $\Lambda \geqslant 1$ such that

$$
\|\nabla \sigma\|_{\infty} \leqslant \Lambda \quad \text { and } \quad \Lambda^{-1}|\xi| \leqslant|\sigma(x) \xi| \leqslant \Lambda|\xi|,
$$

( $\left.\mathrm{A}_{3}\right) b \in C_{b}^{\beta}$ with $\beta>1-\frac{\alpha}{2}$,

(A $\left.\mathrm{A}_{4}\right) \sigma \in C_{b}^{1+\delta}$ for some $\delta>0 . v$ has a compact support and

$$
\operatorname{supp} v \subseteq B_{r_{0}} \text { with } r_{0}=\|\nabla \sigma\|_{\infty}^{-1} \text {. }
$$

The main analytic result of this paper reads as follows.

Theorem 1.1. Let $\alpha \in(0,2)$ and $(1-\alpha)^{+}<\gamma<\beta<1$. Assume $v$ is supported on $B_{R}$ satisfying conditions $\left(\mathrm{A}_{1}\right), \sigma$ satisfies $\left(\mathrm{A}_{2}\right)$ and $b \in C_{b}^{\beta}$. Then there is a constant $\lambda_{0}$ such that for any $\lambda \geqslant \lambda_{0}$ and $f \in C_{b}^{\beta}$, equation (3.1) has a unique solution in $C_{b}^{\alpha+\gamma}$. Moreover,

$$
\lambda\|u\|_{C_{b}^{\gamma}}+\|u\|_{C_{b}^{\alpha+\gamma}} \leqslant C\|f\|_{C_{b}^{\beta}},
$$

where the constants $\lambda_{0}, C$ only depend on $d, \alpha, \beta, \gamma, R, c_{0}, \rho, \Lambda$ and $\|b\|_{C_{b}^{\beta}}$.

Remark 1.2. (1) The assumption on $\sigma$ in Theorem 1.1 can be weakened, but we do not attempt to do that here, since the above results are enough for our main purpose. 
(2) Suppose $\sigma$ is independent of $x$. Assume $v$ only satisfies $\left(\mathrm{A}_{1}\right)$ (does not need to be compactly supported) and $b \in C_{b}^{\beta}$ with $\beta \in\left(1-\frac{\alpha}{2}, 1\right)$. Then by Lemma 3.4 below and the last part of proof for Theorem 3.8, one can see that for any $\gamma \in\left((1-\alpha)^{+}, \beta\right)$ there is a constant $\lambda_{0}$ such that for any $\lambda \geqslant \lambda_{0}$ and $f \in C_{b}^{\beta}$, equation (3.1) has a solution $u \in C_{b}^{\alpha+\gamma}$. Moreover, the estimate (1.5) still holds.

Thanks to the mentioned Zvonkin's transformation, the above result can be applied to prove the regularity properties of solutions to stochastic differential equation (1.1).

Theorem 1.3. Under conditions $\left(\mathrm{A}_{1}\right),\left(\mathrm{A}_{2}\right)$ and $\left(\mathrm{A}_{3}\right)$, for each starting point $x \in \mathbb{R}^{d}$, there is a unique strong solution $X_{t}(x)$ to equation (1.1). Moreover,

(i) if the jumping size of $Z_{t}$ is bounded, then for each $t \geqslant 0, X_{t}(x)$ is Malliavin differentiable;

(ii) if $\sigma$ and $v$ satisfy condition $\left(\mathrm{A}_{4}\right)$ or $\sigma$ does not depend on $x$, then $\left\{X_{t}(x)\right\}_{t \geqslant 0 ; x \in \mathbb{R}^{d}}$ forms $a$ $C^{1}$-stochastic diffeomorphism flow.

Remark 1.4. (1) The strong well-posedness of (1.1) has been essentially established in [CZZ21], so the main contribution of this work lies in Theorem 1.3 (i) and (ii).

(2) All the above results are uniform for all truncated non-degenerate symmetric $\alpha$-stable like processes with $\alpha \in(0,2)$ whose Lévy measure can be singular w.r.t. the Lebesgue measure on $\mathbb{R}^{d}$ (one typical example of $Z$ is the truncated cylindrical $\alpha$-stable process). Moreover, our condition on $b$ is strictly weaker than that in [HP14].

(3) For the existence of $C^{1}$-stochastic diffeomorphism flow, the compactly supported assumption on $v$ is not needed when $\sigma$ is independent of $x$.

To summarize, this article offers the following novel contributions

- in aspect of nonlocal partial differential equations, both $L^{p}$ type and Schauder type estimates are obtained for equation (1.2), where the Lévy measure $v$ associated to the Lévy type operator $\mathscr{L}$ can be singular w.r.t. the Lebesgue measure on $\mathbb{R}^{d}$;

- in aspect of stochastic analysis, regularity of stochastic flows for jump SDEs with irregular coefficients driven by multiplicative noises is studied under very mild assumptions.

1.2. Background and main approach. In the past two decades, there is a great interest for both analysts and probabilists to study nonlocal Lévy type operators and corresponding Markov processes. Parts of the reasons lie in the fact that they have many applications in mathematical finance, control, physics, image processing, etc., and have various connections with other branches of mathematics. Interested readers are referred to [BSW13, JS01, Jac05, SS10] and the references therein for the connection between pseudo-differential operators and Lévy type processes, [BL02a, BL02b, BK05, CK03], etc. for the study of potential theory of nonlocal operators via probabilistic approach and [CCV11, Kas09, Sil06], etc. for Hölder regularity estimates of solutions to nonlocal PDEs.

For the regularity estimates for nonlocal PDEs with first order terms, to our best knowledge, it was Silvestre [Sil12] who first obtained a Schauder type estimate for the parabolic analogy of (1.2) when $\mathscr{L}$ is the usual fractional Laplacian $\Delta^{\alpha / 2}:=-(-\Delta)^{\alpha / 2}$ with $\alpha \in(0,1)$ and $b \in C_{b}^{\beta}$ with $\beta \in(1-\alpha, 1)$. Around the same time, (1.2) was also studied by Priola [Pri12, Pri15] in Hölder spaces for $\alpha$-stable 
like operators with $\alpha \in[1,2)$. The reader can also refer to [dRMP20, DJZ18, DK13, HWW20, LZ19] for some important extensions and [ZZ18] for the Dirichlet problem associated with $\mathscr{L}+b \cdot \nabla$ in the parabolic setting. However, we emphasize that all the above literatures can not cover the important case $\mathscr{L}=\sum_{i=1}^{d}-\left(-\partial_{i i}^{2}\right)^{\alpha / 2}$ with $\alpha \in(0,1 / 2]$. In this paper, we give a systematic and self-contained approach to the apriori estimates for the solutions of resolvent equations, which allows one to handle a large class of Lévy type operator in a uniform way, in particular, for operators associated with cylindrical $\alpha$-stable processes with $\alpha \in(0,2)$. Since $\mathscr{L}$ is not the dominant term when $\alpha \in(0,1)$, it is very hard to apply the perturbation arguments as in the case $\alpha \in(1,2)$. To overcome this difficulty, we resort to some techniques in harmonic analysis, for instance, Littlewood-Paley decomposition and Bernstein type inequalities. To be specific, when $\sigma$ is a matrix, we first give a key Bernstein type inequality (3.2) with a self-contained proof. Then we use the energy method to establish an apriori estimate for the solution of (1.2) in the Sobolev-Slobodeckij space $W_{p}^{s}$, see Lemma 3.4. For the general case with varying coefficient $\sigma$, under the additional condition that $v$ is compactly supported, we also obtain the corresponding conclusion by the usual freezing coefficients method. Using a localization technique from [ZZ21] and [XXZZ20], we then get the desired Schauder type estimate. We need to point out that in [CZZ21], Chen, Zhang and the author of this paper adopted the similar approach of this paper to study the Kolmogorov equation in Besov space $B_{p, \infty}^{s}$. But the main analytic result (Theorem 3.6) therein needs a special assumption on the oscillation of $\sigma$, since $B_{p, \infty}^{s}$ does not enjoy the localization principle (see Lemma 3.6 below). This prevents us from applying their results to our probabilistic purpose.

One the other hand, the study of SDEs driven by non-degenerate Gaussian noises with irregular coefficients has a long history and the literature on this topic is quite impressive. After Zvonkin's pioneering work [Zvo74], there have been a lot of works devoted to studying the strong well-posedness of singular SDEs, among which, we quote [KR05, Ver80, Zha11, Zha16]. Moreover, there are also many articles investigating the regularity properties of $X_{t}(x, \omega)$ w.r.t. $x$ and $\omega$. Among all, let us mention that the weak differentiability of $X_{t}(x)$ w.r.t. $x$ in the multiplicative noise case was shown in [Zha11, Zha16] by using Zvonkin's idea. In [MPMBN ${ }^{+}$13, MBP10, MNP15], the authors adopted another approach based on Malliavin calculus to study the solvability of (1.1) as well as the Malliavin differentiability of the solutions in the additive noise case.

When the driven noise $Z$ is an $\alpha$-stable process, Tanaka, Tsuchiya and Watanabe [TTW74] proved that if $d=1, \sigma=1, Z$ is a symmetric $\alpha$-stable process with $\alpha \in[1,2)$ and $b$ is bounded measurable, then pathwise uniqueness holds for SDE (1.1). They further show that when $\alpha \in(0,1)$, even if $b$ is $\beta$-Hölder continuous with $\beta \in(0,1-\alpha)$, the pathwise uniqueness may fail. For multidimensional case, when $Z$ is an $\alpha$-stable like process with $\alpha \in[1,2)$ and $b$ is $\beta$-Hölder continuous with $\beta>1-\alpha / 2$, the well-posedness of (1.1) was studied by Priola [Pri12, Pri15], see also [Zha13] for the case when $b$ is in some Sobolev spaces. Later, the well-posedness in the supercritical case $(\alpha \in(0,1))$ was studied in [CSZ18, CZZ21]. Weak differentiability of $X_{t}(x)$ w.r.t. $x$ together with the stochastic flow property were also discussed in [CSZ18], when $\sigma=\mathbb{I}$ and the semigroup of $Z$ fulfills some special gradient estimates. Using Malliavin's calculus for jump processes, Haadem and Proske [HP14] studied the strong existence and Malliavin differentiability by a similar approach as 
in $\left[\mathrm{MPMBN}^{+} 13, \mathrm{MBP} 10\right]$. However, they had to assume that $Z_{t}$ is a truncated rotational symmetric $\alpha$-stable process with $\alpha>1, \sigma=\mathbb{I}$ and $b \in C_{b}^{\beta}$ with $\beta>2-\alpha$, which are much stronger than our assumptions. For the weak well-posedness of (1.1), the existence and continuity of the transition probability density of the corresponding Markov process, the readers are referred to [Kul19, Zha19]. We also note that [HL18, KS19, MX18] study the convergence of the Euler-Maruyama approximation for (1.1).

The rest of this paper is organized as follows: In Section 2, we introduce some concepts and facts from Littlewood-Paley theory as well as Malliavin calculus for Lévy processes. In Section 3, we study the nonlocal resolvent equation (1.2) and obtain some apriori estimates in both localized Sobolev spaces and Hölder spaces. In Section 4, we prove our probabilistic results by using our analytic results proved in Section 3 and Zvonkin's transform.

\section{PRELIMINARY}

In this section, we first introduce the (localized) fractional Sobolev spaces and Besov spaces, which are the main function spaces to solve the PDE (1.2) in this paper. For the sake of completeness, we then present some concepts and results about the Malliavin calculus for Lévy processes.

2.1. (Localized) Sobolev spaces and Besov spaces. Let $\chi: \mathbb{R}^{d} \rightarrow[0,1]$ be a smooth radial function satisfying

$$
\chi(\xi)=1,|\xi| \leqslant 1, \chi(\xi)=0,|\xi| \geqslant 3 / 2
$$

Define

$$
\chi_{z}(x):=\chi(x-z)
$$

Definition 2.1. For $n \in \mathbb{N}, s \geqslant 0$ and $p \in(1, \infty)$,

(1) the usual Sobolev space is defined as

$$
W_{p}^{n}:=\left\{f \in L^{p}:\|f\|_{W_{p}^{n}}:=\sum_{k=0}^{n}\left\|\nabla^{k} f\right\|_{p}<\infty\right\} ;
$$

(2) the Sobolev-Slobodeckij semi-norm is defined by

$$
[f]_{\theta, p}:=\left(\iint_{\mathbb{R}^{d} \times \mathbb{R}^{d}} \frac{|f(x)-f(y)|^{p}}{|x-y|^{\theta p+d}} \mathrm{~d} x d y\right)^{\frac{1}{p}}, \theta \in(0,1)
$$

and the Sobolev-Slobodeckij space $W_{p}^{s}$ is defined as

$$
W_{p}^{s}:=\left\{f \in W_{p}^{\lfloor s\rfloor}:\|f\|_{W_{p}^{s}}:=\|f\|_{W_{p}^{\lfloor s\rfloor}}+\sup _{|\alpha|=\lfloor s\rfloor}\left[\partial^{\alpha} f\right]_{s-\lfloor s\rfloor, p}<\infty\right\}, s \notin \mathbb{N} ;
$$

(3) the usual Bessel potential space $H_{p}^{s}$ is defined as

$$
H_{p}^{s}:=\left\{f \in L^{p}:\|f\|_{H_{p}^{s}}:=\left\|(\mathbb{I}-\Delta)^{s / 2} f\right\|_{p}<\infty\right\} .
$$

Now we can define the localized fractional Sobolev space, which will play a crucial role in our study of (1.2). 
Definition 2.2. For $s \geqslant 0, p \in(1, \infty)$, define

$$
\mathscr{W}_{p}^{s}:=\left\{f \in W_{p, l o c}^{s}:\|f\|_{\mathscr{W}_{p}^{s}}:=\sup _{z \in \mathbb{R}^{d}}\left\|f \chi_{z}\right\|_{W_{p}^{s}}<\infty\right\} .
$$

Next we recall some basic facts of the Littlewood-Paley theory and give the definition of Besov spaces. Let $\mathscr{S}\left(\mathbb{R}^{d}\right)$ be the Schwartz space of all rapidly decreasing functions, and $\mathscr{S}^{\prime}\left(\mathbb{R}^{d}\right)$ be the dual space of $\mathscr{S}\left(\mathbb{R}^{d}\right)$ called Schwartz generalized function (or tempered distribution) space. Given $f \in \mathscr{S}\left(\mathbb{R}^{d}\right)$, let $\mathscr{F} f=\hat{f}$ be the Fourier transform of $f$ defined by

$$
\hat{f}(\xi):=(2 \pi)^{-d / 2} \int_{\mathbb{R}^{d}} \mathrm{e}^{-\mathrm{i} \xi \cdot x} f(x) \mathrm{d} x .
$$

Define

$$
\varphi(\xi):=\chi(\xi)-\chi(2 \xi) .
$$

It is easy to see that $\varphi \geqslant 0$ and $\operatorname{supp} \varphi \subset B_{3 / 2} \backslash B_{1 / 2}$ and

$$
\chi(2 \xi)+\sum_{j=0}^{k} \varphi\left(2^{-j} \xi\right)=\chi\left(2^{-k} \xi\right) \stackrel{k \rightarrow \infty}{\rightarrow} 1 .
$$

In particular, if $\left|j-j^{\prime}\right| \geqslant 2$, then

$$
\operatorname{supp} \varphi\left(2^{-j} \cdot\right) \cap \operatorname{supp} \varphi\left(2^{-j^{\prime}} \cdot\right)=\varnothing .
$$

Let $h:=\mathscr{F}^{-1} \chi$ be the inverse Fourier transform of $\chi$. Define

$$
h_{-1}(x):=\mathscr{F}^{-1} \chi(2 \cdot)(x)=2^{-d} h\left(2^{-1} x\right) \in \mathscr{S}\left(\mathbb{R}^{d}\right),
$$

and for $j \geqslant 0$,

$$
h_{j}(x):=\mathscr{F}^{-1} \varphi\left(2^{-j} \cdot\right)(x)=2^{j d} h\left(2^{j} x\right)-2^{(j-1) d} h\left(2^{j-1} x\right) \in \mathscr{S}\left(\mathbb{R}^{d}\right) .
$$

Operators $\Delta_{j}$ and $S_{j}$ is defined by

$$
\Delta_{j} f:=\left\{\begin{array}{c}
\mathscr{F}^{-1}(\chi(2 \cdot) \mathscr{F} f), j=-1, \\
\mathscr{F}^{-1}\left(\varphi\left(2^{-j} \cdot\right) \mathscr{F} f\right), j \geqslant 0,
\end{array} \quad S_{j} f:=\sum_{k \leqslant j-1} \Delta_{k} f .\right.
$$

It is easy to see that

$$
\Delta_{j} f(x)=\left(h_{j} * f\right)(x)=\int_{\mathbb{R}^{d}} h_{j}(x-y) f(y) \mathrm{d} y, j \geqslant-1 .
$$

Definition 2.3. For $s \in \mathbb{R}$ and $p, q \in[1, \infty]$, the Besov space $B_{p, q}^{s}$ is defined as the set of all $f \in \mathscr{S}^{\prime}\left(\mathbb{R}^{d}\right)$ with

$$
\|f\|_{B_{p, q}^{s}}:=\left(\sum_{j \geqslant-1} 2^{j s q}\left\|\Delta_{j} f\right\|_{p}^{q}\right)^{1 / q}<\infty .
$$

Assume that $k \in \mathbb{N}, \beta \in[0,1)$ and $s=k+\alpha$, let $C_{b}^{s}$ denote the collection of all bounded functions such that

$$
\|f\|_{C_{b}^{s}}:=\sum_{i=0}^{k}\left\|\nabla^{k} f\right\|_{\infty}+\sup _{x \neq y} \frac{\left|\nabla^{k} f(x)-\nabla^{k} f(y)\right|}{|x-y|^{\beta}}<\infty .
$$


Last, we present two Bernstein type inequalities and the interrelationships of the above function spaces. The following two lemmas can be found in [Tri92].

Lemma 2.4. Let $1 \leqslant p \leqslant q \leqslant \infty$ and $j \geqslant 0$. For any $f \in \mathscr{S}^{\prime}\left(\mathbb{R}^{d}\right)$ with $\Delta_{j} f \in L^{p}$, we have

$$
\left\|\nabla^{k} \Delta_{j} f\right\|_{q} \leqslant C_{p} 2^{\left(k+d\left(\frac{1}{p}-\frac{1}{q}\right)\right) j}\left\|\Delta_{j} f\right\|_{p}, k=0,1, \cdots,
$$

and

$$
\left\|(-\Delta)^{s / 2} \Delta_{j} f\right\|_{q} \leqslant C_{p} 2^{\left(s+d\left(\frac{1}{p}-\frac{1}{q}\right)\right) j}\left\|\Delta_{j} f\right\|_{p}, s \in \mathbb{R} .
$$

Lemma 2.5. Let $s>\varepsilon>0$ and $p \in(1, \infty)$.

(1) It holds that $H_{p}^{s+\varepsilon} \subseteq W_{p}^{s} \subseteq H_{p}^{s-\varepsilon}$.

(2) If $0<s-\frac{d}{p} \notin \mathbb{N}$, then $H_{p}^{s} \subseteq C_{b}^{s-\frac{d}{p}}$ and $W_{p}^{s} \subseteq C_{b}^{s-\frac{d}{p}}$.

(3) If $0<s \notin \mathbb{N}$, then $B_{\infty, \infty}^{s}=C_{b}^{s}$ and $B_{p, p}^{s}=W_{p}^{s}$.

The following lemma reveals the relationship between $\mathscr{W}_{p}^{s}$ and $C_{b}^{s}$.

Lemma 2.6. (1) For any $\gamma>d / p$, we have $\mathscr{W}_{p}^{\gamma} \subseteq C_{b}^{\gamma-\frac{d}{p}}$;

(2) For any $\beta>\gamma \geqslant 0$, we have $C_{b}^{\beta} \subseteq \mathscr{W}_{p}^{\gamma}$.

Proof. The first conclusion is just a consequence of Sobolev embedding theorem. We only need to prove the second conclusion when $0 \leqslant \gamma<\beta<1$. Obviously, if $u \in C_{b}^{\beta}$, then

$$
\left\|u \chi_{z}\right\|_{p} \lesssim\|u\|_{L^{\infty}} \lesssim\|u\|_{C_{b}^{\beta}}
$$

where $\chi_{z}$ is defined by (2.1). Hence, $C_{b}^{\beta} \subseteq \mathscr{W}_{p}^{0}$. Note $u \chi_{z}(x)=u \chi_{z}(y)=0$ if $y \in B_{1}(x)$ and $x \notin B_{5 / 2}(z)$. So for any $0<\gamma<\beta<1$, we have

$$
\begin{aligned}
& \iint_{\mathbb{R}^{d} \times \mathbb{R}^{d}} \frac{\left|u \chi_{z}(x)-u \chi_{z}(y)\right|^{p}}{|x-y|^{d+\gamma p}} \mathrm{~d} x \mathrm{~d} y \\
& \lesssim \iint_{|x-z| \leqslant \frac{5}{2},|x-y| \leqslant 1} \frac{\left|u \chi_{z}(x)-u \chi_{z}(y)\right|^{p}}{|x-y|^{d+\gamma p}} \mathrm{~d} x \mathrm{~d} y+\iint_{|x-y|>1} \frac{\left|u \chi_{z}(x)-u \chi_{z}(y)\right|^{p}}{|x-y|^{d+\gamma p}} \mathrm{~d} x \mathrm{~d} y \\
& \lesssim \iint_{|x-z| \leqslant \frac{5}{2},|x-y| \leqslant 1} \frac{\left|\chi_{z}(x)\right|^{p} \cdot|u(x)-u(y)|^{p}}{|x-y|^{d+\gamma p}} \mathrm{~d} x \mathrm{~d} y \\
& +\iint_{|x-z| \leqslant \frac{5}{2},|x-y| \leqslant 1} \frac{|u(y)|^{p} \cdot\left|\chi_{z}(x)-\chi_{z}(y)\right|^{p}}{|x-y|^{d+\gamma p}} \mathrm{~d} x \mathrm{~d} y \\
& +\iint_{|x-y|>1} \frac{\left|u \chi_{z}(x)\right|^{p}+\left|u \chi_{z}(y)\right|^{p}}{|x-y|^{d+\gamma p}} \mathrm{~d} x \mathrm{~d} y \\
& \lesssim \iint_{|x-z| \leqslant \frac{5}{2},|x-y| \leqslant 1} \frac{\|u\|_{C_{b}^{\beta}}^{p}|x-y|^{\beta p}}{|x-y|^{d+\gamma p}} \mathrm{~d} x \mathrm{~d} y+\iint_{|x-y|>1} \frac{\left|u \chi_{z}(x)\right|^{p}}{|x-y|^{d+\gamma p}} \mathrm{~d} x \mathrm{~d} y \\
& \lesssim\|u\|_{C_{b}^{\beta}}^{p} \int_{|x-z| \leqslant \frac{5}{2}} \mathrm{~d} x \int_{|w| \leqslant 1}|w|^{-d+(\beta-\gamma) p} \mathrm{~d} w+\|u\|_{L^{\infty}}^{p} \int_{|x-z| \leqslant \frac{3}{2}} \mathrm{~d} x \int_{|w|>1}|w|^{-d-\gamma p} \mathrm{~d} w \\
& \lesssim\|u\|_{C_{b}^{\beta}}^{p} \text {. }
\end{aligned}
$$


This yields, $\sup _{z \in \mathbb{R}^{d}}\left\|u \chi_{z}\right\|_{W_{p}^{\gamma}} \lesssim C\|u\|_{C_{b}^{\beta}}$. So we complete our proof.

2.2. Malliavin Derivate for Lévy processes. In this subsection, we introduce some basic concepts of Malliavin calculus for Lévy processes. The reader can refer to [DNØP09] for more details.

Suppose $N(\mathrm{~d} t, \mathrm{~d} x)$ is a Poisson point process with intensity measure $v(\mathrm{~d} z)$. Let $\left\{\mathscr{F}_{t}\right\}_{0 \leqslant t \leqslant T}$ be the filtration generated by $N$ and $\widetilde{N}(\mathrm{~d} t, \mathrm{~d} z):=N(\mathrm{~d} t, \mathrm{~d} z)-v(\mathrm{~d} z) \mathrm{d} t$.

For each $n \in \mathbb{N}_{+}$and $f \in L^{2}\left(\left([0, T] \times \mathbb{R}^{d}\right)^{n} ;(\lambda \times v)^{n}\right)$, define

$$
\widetilde{f}\left(t_{1}, z_{1} ; \cdots ; t_{n}, z_{n}\right):=\frac{1}{n !} \sum_{\sigma \in S_{n}} f\left(t_{\sigma(1)}, z_{\sigma(1)} ; \cdots ; t_{\sigma(n)}, z_{\sigma(n)}\right) .
$$

The collection of all square integrable symmetric functions is denoted by $\widetilde{L}^{2}\left(\left([0, T] \times \mathbb{R}^{d}\right)^{n},(\lambda \times\right.$ $\left.v)^{n}\right)$ (abbreviated by $\left.\widetilde{L}^{2}\left((\lambda \times v)^{n}\right)\right)$. For any $n \in \mathbb{N}_{+}$and $f_{n} \in \widetilde{L}^{2}\left((\lambda \times v)^{n}\right)$, we set

$$
I_{n}\left(f_{n}\right):=\int_{\left([0, T] \times \mathbb{R}^{d}\right)^{n}} f_{n}\left(t_{1}, z_{1} ; \cdots ; t_{n}, z_{n}\right) \widetilde{N}^{\otimes n}(\mathrm{~d} \mathbf{t}, \mathrm{d} \mathbf{z}), \mathbf{t}=\left(t_{1}, \cdots t_{n}\right), \mathbf{z}=\left(z_{1}, \cdots, z_{n}\right)
$$

and $I_{0}\left(f_{0}\right):=f_{0}$ for the constant values $f_{0} \in \mathbb{R}$.

Definition 2.7. The stochastic Sobolev space $\mathbb{D}_{2}^{1}$ consists of all $\mathscr{F}_{T}$ measurable random variables $F \in L^{2}(\mathbf{P})$ with chaos expansion $F=\sum_{n=0}^{\infty} I_{n}\left(f_{n}\right)$ satisfying

$$
\sum_{n=1}^{\infty} n n !\left\|f_{n}\right\|_{L^{2}\left((\lambda \times v)^{n}\right)}^{2}<\infty .
$$

Define the Malliavin derivative operator $D: \mathbb{D}_{2}^{1} \ni F \mapsto D F \in L^{2}(\lambda \times v \times \mathbf{P})$ by

$$
D_{t, z} F:=\sum_{n=1}^{\infty} n I_{n-1}\left(\widetilde{f}_{n}(\cdot ; t, z)\right), \quad F \in \mathbb{D}_{2}^{1}
$$

where $\widetilde{f}_{n}(\cdot ; t, z)$ is defined as 2.6.

Note that

$$
\|D F\|_{L^{2}((\lambda \times v \times \mathbf{P}))}^{2}=\sum_{n=1}^{\infty} n n !\left\|f_{n}\right\|_{L^{2}\left((\lambda \times v)^{n}\right)}^{2} .
$$

Thus, $F \in \mathbb{D}_{2}^{1}$ if and only if $F \in L^{2}(\mathbf{P})$ and $D F \in L^{2}((\lambda \times v \times \mathbf{P}))$.

The next lemma about the closability of Malliavin derivative is a consequence of [DNØP09, Theorem 12.6].

Lemma 2.8. Assume $F_{n} \in \mathbb{D}_{2}^{1}, F_{n} \rightarrow F$ in $L^{2}(\mathbf{P})$ and

$$
\sup _{n}\left\|D F_{n}\right\|_{L^{2}(\lambda \times v \times \mathbf{P})} \leqslant M<\infty .
$$

Then, $F \in \mathbb{D}_{2}^{1}$ and

$$
\|D F\|_{L^{2}((\lambda \times v \times \mathbf{P}))} \leqslant M .
$$


Proof. By our assumption, $\left\{D F_{n}\right\}_{n \in \mathbb{N}}$ is bounded in $L^{2}(\lambda \times v \times \mathbf{P})$, thus Banach-Saks theorem implies that, the Cesàro mean sequence of a suitable subsequence of $\left\{D F_{n}\right\}$, say $\left\{D F_{n_{k}}\right\}$, converges strongly to some $G \in L^{2}(\lambda \times v \times \mathbf{P})$, i.e.

$$
D\left(\frac{1}{m} \sum_{k=1}^{m} F_{n_{k}}\right)=\frac{1}{m} \sum_{k=1}^{m} D F_{n_{k}} \rightarrow G, \text { in } L^{2}(\lambda \times v \times \mathbf{P}) .
$$

On the other hand, $\frac{1}{m} \sum_{k=1}^{m} F_{n_{k}} \rightarrow F$ in $L^{2}(\mathbf{P})$, by [DNØP09, Theorem 12.6], we get $F \in \mathbb{D}_{2}^{1}, D F=G$ and

$$
\begin{gathered}
\|D F\|_{L^{2}((\lambda \times v \times \mathbf{P}))}=\lim _{m \rightarrow \infty} \frac{1}{m}\left\|\sum_{k=1}^{m} D F_{n_{k}}\right\|_{L^{2}((\lambda \times v \times \mathbf{P}))} \\
\leqslant \liminf _{m \rightarrow \infty} \frac{1}{m} \sum_{k=1}^{m}\left\|D F_{n_{k}}\right\|_{L^{2}((\lambda \times v \times \mathbf{P}))} \leqslant M .
\end{gathered}
$$

\section{A STUDY OF NONLOCAL PARABOLIC EQUATIONS}

In this section we study the solvability and regularity of nonlocal elliptic equations with first order terms. First of all, we introduce the nonlocal operator studied in this work. Given a Borel measurable function $\sigma: \mathbb{R}^{d} \rightarrow \mathbb{R}^{d} \otimes \mathbb{R}^{d}$, we define

$$
\mathscr{L} f(x)=L_{\sigma} f(x):=\int_{\mathbb{R}^{d}}\left(f(x+\sigma(x) z)-f(x)-\nabla f(x) \cdot \sigma(x) z \mathbf{1}_{B_{1}}(z)\right) v(\mathrm{~d} z) .
$$

When $\sigma(x)=\sigma$ is a matrix, by Fourier's transform, we have

$$
\widehat{L_{\sigma} f}(\xi)=-\psi_{\sigma}(\xi) \hat{f}(\xi)
$$

where the symbol $\psi_{\sigma}(\xi)$ takes the form

$$
\psi_{\sigma}(\xi)=-\int_{\mathbb{R}^{d}}\left(\mathrm{e}^{\mathrm{i} \xi \cdot \sigma z}-1-\mathrm{i} \sigma z \mathbf{1}_{B_{1}}(z) \cdot \xi\right) v(\mathrm{~d} z) .
$$

Next we consider the following equation:

$$
\lambda u-\mathscr{L} u-b \cdot \nabla u=f, \lambda>0 .
$$

3.1. Constant coefficient case: $\sigma(x)=\sigma$. In this subsection we consider equation (3.1) with nondegenerate constant coefficient $\sigma(x)=\sigma \in \mathbb{R}^{d \times d}$. First of all, we establish the following Bernstein type inequality for nonlocal operator $L_{\sigma}$, which will play a crucial role in the sequel. Recall that $\Delta_{j}$ is the dyadic block operator defined in section 2 .

Lemma 3.1. Let $p \geqslant 2$ and $j=-1,0,1, \cdots$. Assume that $v$ satisfies (1.3) and $f \in \mathscr{S}^{\prime}\left(\mathbb{R}^{d}\right)$ with $\Delta_{j} f \in L^{p}$, then

$$
\int_{\mathbb{R}^{d}}\left|\Delta_{j} f\right|^{p-2} \Delta_{j} f L_{\sigma} \Delta_{j} f \mathrm{~d} x \leqslant 0
$$


Moreover, there are constants $c>0, j_{0} \in \mathbb{N}$ only depending on $d, p, c_{0}, \rho$ and $\Lambda$ such that for any $j=j_{0}, j_{0}+1, j_{0}+2, \cdots$,

$$
-\int_{\mathbb{R}^{d}}\left|\Delta_{j} f\right|^{p-2} \Delta_{j} f L_{\sigma} \Delta_{j} f \mathrm{~d} x \geqslant c 2^{\alpha j}\left\|\Delta_{j} f\right\|_{p}^{p}
$$

Proof. Without loss of generality, we can assume $\sigma=\mathbb{I}$ and $f, g \in \mathscr{S}\left(\mathbb{R}^{d}\right)$. Denote $L=L_{\mathbb{I}}$ and $\langle f, g\rangle:=\int_{\mathbb{R}^{d}} f(x) g(x) \mathrm{d} x$. By the symmetry of $v$, we have

$$
\langle f,-L g\rangle=\frac{1}{2} \int_{\mathbb{R}^{d} \times \mathbb{R}^{d}}(f(x+z)-f(x))(g(x+z)-g(x)) v(\mathrm{~d} z) \mathrm{d} x .
$$

By (3.3) and the following elementary inequality:

$$
p(a-b)\left(|a|^{p-2} a-|b|^{p-2} b\right) \geqslant\left(a|a|^{\frac{p}{2}-1}-b|b|^{\frac{p}{2}-1}\right)^{2}, \forall p \geqslant 2, a, b \in \mathbb{R},
$$

we get,

$$
\begin{aligned}
& \left\langle f|f|^{p-2},-L f\right\rangle \\
= & \frac{1}{2} \int_{\mathbb{R}^{d} \times \mathbb{R}^{d}}(f(x+z)-f(x))\left(|f|^{p-2} f(x+z)-|f|^{p-2} f(x)\right) v(\mathrm{~d} z) \mathrm{d} x \\
\geqslant & \frac{1}{2 p} \int_{\mathbb{R}^{d} \times \mathbb{R}^{d}}\left(f|f|^{\frac{p}{2}-1}(x+z)-f|f|^{\frac{p}{2}-1}(x)\right)^{2} v(\mathrm{~d} z) \mathrm{d} x \\
= & \frac{1}{p}\left\langle f|f|^{\frac{p}{2}-1},-L\left(f|f|^{\frac{p}{2}-1}\right)\right\rangle=\frac{1}{p} \int_{\mathbb{R}^{d}}\left|(-L)^{\frac{1}{2}}\left(f|f|^{\frac{p}{2}-1}\right)\right|^{2} \mathrm{~d} x .
\end{aligned}
$$

This implies our first desired inequality. Noting that for all $x \in[0,1], 1-\cos x \geqslant c x^{2}$, by $\left(\mathrm{A}_{1}\right)$, for any $|\xi|>\rho^{-1}$,

$$
\begin{aligned}
\psi(\xi) & =\int_{\mathbb{R}^{d}}(1-\cos (z \cdot \xi)) v(\mathrm{~d} z) \geqslant c \int_{|z| \leqslant|\xi|^{-1}}|z \cdot \xi|^{2} v(\mathrm{~d} z) \\
& \gtrsim|\xi|^{2} \int_{|z| \leqslant|\xi|^{-1}}\left|z \cdot \frac{\xi}{|\xi|}\right|^{2} v(\mathrm{~d} z) \stackrel{(1.3)}{\gtrsim}|\xi|^{\alpha} .
\end{aligned}
$$

On the other hand, it is easy to see that

$$
\psi(\xi) \gtrsim|\xi|^{2}, \quad \forall|\xi| \leqslant \rho^{-1} .
$$

Thus, $\psi(\xi) \gtrsim|\xi|^{\alpha}-1$. Noting that $\mathscr{F}\left((-L)^{1 / 2} g\right)(\xi)=\sqrt{\psi(\xi)} \mathscr{F}(g)(\xi)$, by Plancherel formula,

$$
\begin{aligned}
& \int_{\mathbb{R}^{d}}\left|(-L)^{\frac{1}{2}}\left(f|f|^{\frac{p}{2}-1}\right)\right|^{2} \mathrm{~d} x=\int_{\mathbb{R}^{d}} \psi(\xi)\left|\mathscr{F}\left(f|f|^{\frac{p}{2}-1}\right)(\xi)\right|^{2} \mathrm{~d} \xi \\
\geqslant & c \int_{\mathbb{R}^{d}}|\xi|^{\alpha}\left|\mathscr{F}\left(f|f|^{\frac{p}{2}-1}\right)(\xi)\right|^{2} \mathrm{~d} \xi-C \int_{\mathbb{R}^{d}}\left|\mathscr{F}\left(f|f|^{\frac{p}{2}-1}\right)(\xi)\right|^{2} \mathrm{~d} \xi \\
\geqslant & c \int_{\mathbb{R}^{d}}\left|(-\Delta)^{\frac{\alpha}{4}}\left(f|f|^{\frac{p}{2}-1}\right)\right|^{2} \mathrm{~d} x-C \int_{\mathbb{R}^{d}}|f|^{p} \mathrm{~d} x .
\end{aligned}
$$

Combing (3.4), (3.5) and using the elementary inequality:

$$
\left.|a| a\right|^{\frac{p}{2}-1}-\left.b|b|^{\frac{p}{2}-1}\right|^{2} \geqslant c_{p}|a-b|^{p}, \forall a, b \in \mathbb{R}, p \geqslant 2,
$$


we obtain

$$
\begin{aligned}
\int_{\mathbb{R}^{d}} f|f|^{p-2}(-L f) \mathrm{d} x & \geqslant c \int_{\mathbb{R}^{d}}\left|(-\Delta)^{\frac{\alpha}{4}}\left(f|f|^{\frac{p}{2}-1}\right)\right|^{2} \mathrm{~d} x-C \int_{\mathbb{R}^{d}}|f|^{p} \mathrm{~d} x \\
& \geqslant c \int_{\mathbb{R}^{d} \times \mathbb{R}^{d}} \frac{\left.|f| f\right|^{\frac{p}{2}-1}(x)-\left.f|f|^{\frac{p}{2}-1}(y)\right|^{2}}{|x-y|^{d+\alpha}} \mathrm{d} x \mathrm{~d} y-C\|f\|_{p}^{p} \\
& \geqslant c \int_{\mathbb{R}^{d} \times \mathbb{R}^{d}} \frac{|f(x)-f(y)|^{p}}{|x-y|^{d+\frac{\alpha}{p} \cdot p}} \mathrm{~d} x \mathrm{~d} y-C\|f\|_{p}^{p}=c[f]_{\frac{\alpha}{p}, p}^{p}-C\|f\|_{p}^{p} .
\end{aligned}
$$

Noting that $\frac{\alpha}{p} \leqslant \frac{\alpha}{2}<1$, by Theorem 2.36 of [BCD11], for any $j \geqslant 0$,

$$
\left[\Delta_{j} f\right]_{\frac{\alpha}{p}, p}^{p}=\|f\|_{\dot{B}_{p, p}^{\frac{\alpha}{p}}}=\sum_{k=-\infty}^{\infty} 2^{\alpha k}\left\|\Delta_{k} \Delta_{j} f\right\|_{p}^{p} \asymp 2^{\alpha j}\left\|\Delta_{j} f\right\|_{p}^{p}
$$

Thus,

$$
-\int_{\mathbb{R}^{d}}\left|\Delta_{j} f\right|^{p-2} \Delta_{j} f L \Delta_{j} f \mathrm{~d} x \geqslant\left(c 2^{\alpha j}-C\right)\left\|\Delta_{j} f\right\|_{p}^{p} .
$$

Letting $j_{0}=1+\log _{2}(C / c) / \alpha$, we get the desired result.

Remark 3.2. Similar result was also proved in [CZZ21] by using inequality (1.5) in [CMZ07]. Our proof presented here is much simpler and self-contained.

Then we present a simple commutator estimate, which is a special case of Lemma 2.3 in [CZZ21].

Lemma 3.3. Let $j \geqslant-1, \beta \in(0,1)$. Assume that $b \in C_{b}^{\beta}$ and $u \in W_{p}^{1}$, then

$$
\left\|\left[\Delta_{j}, b \cdot \nabla\right] u\right\|_{p} \lesssim 2^{-\beta j}\|b\|_{C_{b}^{\beta}}\|\nabla u\|_{p}
$$

Proof. By (2.3), we have

$$
\left[\Delta_{j}, b \cdot \nabla\right] u(x)=\int_{\mathbb{R}^{d}} h_{j}(y)(b(x-y)-b(x)) \cdot \nabla u(x-y) \mathrm{d} y .
$$

For $j \geqslant 0$, by Minkowski 's inequality and (2.2), we have

$$
\begin{aligned}
\left\|\left[\Delta_{j}, b \cdot \nabla\right] u\right\|_{p} & \leqslant \int_{\mathbb{R}^{d}} h_{j}(y)\|b(\cdot-y)-b(\cdot)\|_{\infty}\|\nabla u\|_{p} \mathrm{~d} y \\
& \lesssim\|b\|_{C_{b}^{\beta}}\|\nabla u\|_{p} \int_{\mathbb{R}^{d}}\left|h_{j}(y)\right||y|^{\beta} \mathrm{d} y \\
& =\|b\|_{C_{b}^{\beta}}\|\nabla u\|_{p^{-j}} \int_{\mathbb{R}^{d}}|2 h(2 y)-h(y)||y|^{\beta} \mathrm{d} y \\
& \lesssim 2^{-\beta j}\|b\|_{C_{b}^{\beta}}\|\nabla u\|_{p} .
\end{aligned}
$$

The estimate for $j=-1$ is simpler, so we complete our proof.

Last we state our main result of this subsection. 
Lemma 3.4. Let $\alpha \in(0,2),(1-\alpha)^{+}<\gamma<\beta<1$ and $p \in[2, \infty)$. Suppose that $v$ satisfies (1.3), then there is a constant $\lambda_{0}>0$ such that for all $\lambda \geqslant \lambda_{0}$ and $f \in W_{p}^{\gamma}$, equation (3.1) admits a unique solution $u \in W_{p}^{\alpha+\gamma}$. Moreover,

$$
\lambda\|u\|_{W_{p}^{\gamma}}+\|u\|_{W_{p}^{\alpha+\gamma}} \leqslant C\|f\|_{W_{p}^{\gamma}},
$$

where $\lambda_{0}, C$ depend only on $d, p, \alpha, \beta, \gamma, c_{0}, \rho, \Lambda$ and $\|b\|_{C_{b}^{\beta}}$.

Proof. Thanks to method of continuity, our main task is to show the apriori estimates (3.7). Assume $u \in W_{p}^{\alpha+\gamma}$ satisfies equation (3.1). Using operator $\Delta_{j}$ act on both sides of (3.1) and noticing that $\Delta_{j} \mathscr{L}=\Delta_{j} L_{\sigma}=L_{\sigma} \Delta_{j}=\mathscr{L} \Delta_{j}$, we have

$$
\lambda \Delta_{j} u=\mathscr{L} \Delta_{j} u+\Delta_{j}(b \cdot \nabla u)+\Delta_{j} f .
$$

For $p \geqslant 2$, by the chain rule or multiplying both sides by $\left|\Delta_{j} u\right|^{p-2} \Delta_{j} u$ and then integrating in $x$, we obtain

$$
\begin{aligned}
\lambda \int_{\mathbb{R}^{d}}\left|\Delta_{j} u\right|^{p}= & \int_{\mathbb{R}^{d}}\left|\Delta_{j} u\right|^{p-2} \Delta_{j} u\left[\mathscr{L} \Delta_{j} u+\Delta_{j}(b \cdot \nabla u)+\Delta_{j} f\right] \mathrm{d} x \\
= & \int_{\mathbb{R}^{d}}\left|\Delta_{j} u\right|^{p-2} \Delta_{j} u \mathscr{L} \Delta_{j} u \mathrm{~d} x+\int_{\mathbb{R}^{d}}\left|\Delta_{j} u\right|^{p-2} \Delta_{j} u\left[\Delta_{j}, b \cdot \nabla\right] u \mathrm{~d} x \\
& +\int_{\mathbb{R}^{d}}\left|\Delta_{j} u\right|^{p-2} \Delta_{j} u(b \cdot \nabla) \Delta_{j} u \mathrm{~d} x+\int_{\mathbb{R}^{d}}\left|\Delta_{j} u\right|^{p-2} \Delta_{j} u \Delta_{j} f \mathrm{~d} x \\
= & : I_{j}^{(1)}+I_{j}^{(2)}+I_{j}^{(3)}+I_{j}^{(4)} .
\end{aligned}
$$

For $I_{j}^{(1)}$, recalling $\mathscr{L}=L_{\sigma}$ and by Lemma 3.1, there are constants $c>0$ and $j_{0} \in \mathbb{N}$ such that

$$
I_{j}^{(1)} \leqslant 0, \quad j \geqslant-1 ; \quad I_{j}^{(1)} \leqslant-c 2^{\alpha j}\left\|\Delta_{j} u\right\|_{p}^{p}, \quad j=j_{0}, j_{0}+1, j_{0}+2, \cdots .
$$

For $I_{j}^{(2)}$, using Lemma 3.3 and Hölder's inequality, we have that for all $j=-1,0,1, \cdots$,

$$
\begin{aligned}
I_{j}^{(2)} & \leqslant\left\|\left[\Delta_{j}, b \cdot \nabla\right] u\right\|_{p}\left\|\Delta_{j} u\right\|_{p}^{p-1} \\
& \lesssim 2^{-\beta j}\|b\|_{C_{b}^{\beta}}\|\nabla u\|_{p}\left\|\Delta_{j} u\right\|_{p}^{p-1} .
\end{aligned}
$$

For $I_{j}^{(3)}$, we write

$$
\begin{aligned}
I_{j}^{(3)} & =\int_{\mathbb{R}^{d}}\left(\left(b-S_{j} b\right) \cdot \nabla\right) \Delta_{j} u\left|\Delta_{j} u\right|^{p-2} \Delta_{j} u \mathrm{~d} x \\
& +\int_{\mathbb{R}^{d}}\left(S_{j} b \cdot \nabla\right) \Delta_{j} u\left|\Delta_{j} u\right|^{p-2} \Delta_{j} u \mathrm{~d} x=: I_{j}^{(31)}+I_{j}^{(32)} .
\end{aligned}
$$

For $I_{j}^{(31)}$, by Bernstein's inequality (2.4), we have

$$
\begin{aligned}
I_{j}^{(31)} & \leqslant \sum_{k \geqslant j}\left\|\left(\Delta_{k} b \cdot \nabla\right) \Delta_{j} u\right\|_{p}\left\|\Delta_{j} u\right\|_{p}^{p-1} \\
& \leqslant \sum_{k \geqslant j}\left\|\Delta_{k} b\right\|_{\infty}\left\|\nabla \Delta_{j} u\right\|_{p}\left\|\Delta_{j} u\right\|_{p}^{p-1}
\end{aligned}
$$




$$
\begin{aligned}
& \lesssim 2^{j}\left\|\Delta_{j} u\right\|_{p}^{p} \sum_{k \geqslant j}\left\|\Delta_{k} b\right\|_{\infty} \leqslant 2^{j}\left\|\Delta_{j} u\right\|_{p}^{p}\|b\|_{C_{b}^{\beta}} \sum_{k \geqslant j} 2^{-\beta k} \\
& \lesssim 2^{(1-\beta) j}\|b\|_{C_{b}^{\beta}}\left\|\Delta_{j} u\right\|_{p}^{p} .
\end{aligned}
$$

For $I_{j}^{(32)}$, by integration by parts formula and (2.4) again, we have

$$
\begin{aligned}
I_{j}^{(32)} & =\frac{1}{p} \int_{\mathbb{R}^{d}}\left(S_{j} b \cdot \nabla\right)\left|\Delta_{j} u\right|^{p} \mathrm{~d} x=-\frac{1}{p} \int_{\mathbb{R}^{d}} S_{j} \operatorname{div} b\left|\Delta_{j} u\right|^{p} \mathrm{~d} x \\
& \leqslant \frac{1}{p}\left\|S_{j} \operatorname{div} b\right\|_{\infty}\left\|\Delta_{j} u\right\|_{p}^{p} \leqslant \frac{1}{p} \sum_{k \leqslant j}\left\|\Delta_{k} \operatorname{div} b\right\|_{\infty}\left\|\Delta_{j} u\right\|_{p}^{p} \\
& \lesssim \sum_{k \leqslant j} 2^{k}\left\|\Delta_{k} b\right\|_{\infty}\left\|\Delta_{j} u\right\|_{p}^{p} \\
& \lesssim 2^{(1-\beta) j}\|b\|_{C_{b}^{\beta}}\left\|\Delta_{j} u\right\|_{p}^{p} .
\end{aligned}
$$

Combining the above calculations, we obtain

$$
\begin{aligned}
\lambda\left\|\Delta_{j} u\right\|_{p}^{p}+c 2^{\alpha j} \mathbf{1}_{\left\{j \geqslant j_{0}\right\}}\left\|\Delta_{j} u\right\|_{p}^{p} \leqslant & C 2^{-\beta j}\|b\|_{C_{b}^{\beta}}\|\nabla u\|_{p}\left\|\Delta_{j} u\right\|_{p}^{p-1} \\
& +C 2^{(1-\beta) j}\|b\|_{C_{b}^{\beta}}\left\|\Delta_{j} u\right\|_{p}^{p}+C\left\|\Delta_{j} u\right\|_{p}^{p-1}\left\|\Delta_{j} f\right\|_{p} .
\end{aligned}
$$

By dividing both sides by $\left\|\Delta_{j} u\right\|_{p}^{p-1}$, we get

$$
\begin{aligned}
& \lambda\left\|\Delta_{j} u\right\|_{p}+c 2^{\alpha j} \mathbf{1}_{\left\{j \geqslant j_{0}\right\}}\left\|\Delta_{j} u\right\|_{p}-C 2^{(1-\gamma) j}\|b\|_{C_{b}^{\beta}}\left\|\Delta_{j} u\right\|_{p} \\
\leqslant & C 2^{-\beta j}\|b\|_{C_{b}^{\beta}}\|\nabla u\|_{p}+C\left\|\Delta_{j} f\right\|_{p} .
\end{aligned}
$$

Since $1-\gamma<\alpha$, for some $\lambda$ sufficiently large and all $j \geqslant-1$,

$$
\lambda\left\|\Delta_{j} u\right\|_{p}+2^{\alpha j}\left\|\Delta_{j} u\right\|_{p} \leqslant C 2^{-\beta j}\|\nabla u\|_{p}+C\left\|\Delta_{j} f\right\|_{p} .
$$

Multiplying both sides of (3.8) by $2^{\gamma j}$ and then taking $\ell^{p}$ norm over $j$, we obtain

$$
\lambda\|u\|_{W_{p}^{\gamma}}+\|u\|_{W_{p}^{\alpha+\gamma}} \leqslant C_{1}\left(\|\nabla u\|_{p}+\|f\|_{W_{p}^{\gamma}}\right),
$$

where $C_{1}$ only depends on $d, p, \alpha, \beta, \gamma, c_{0}, \rho$ and $\|b\|_{C_{b}^{\beta}}$. Recalling that $\alpha+\gamma>1$ and using interpolation theorem, we have $\|\nabla u\|_{p} \leqslant \frac{1}{2 C_{1}}\|u\|_{W_{p}^{\alpha+\gamma}}+C^{\prime}\|u\|_{W_{p}^{\gamma}}$. Choosing $\lambda_{0}>2 C_{1} C^{\prime}$, we complete the proof for (3.7).

3.2. Varying coefficient case. In this subsection, we consider the varying coefficient case.

Lemma 3.5. Assume that $\alpha \in(0,2)$ and $v$ satisfies condition (1.4), then for any $r \in(0, \rho)$ and $0 \leqslant \theta^{\prime}<\alpha<\theta$,

$$
\int_{|z| \leqslant r}|z|^{\theta} v(\mathrm{~d} z) \lesssim r^{\theta-\alpha}
$$

and

$$
\int_{r<|z| \leqslant 1}|z|^{\theta^{\prime}} v(\mathrm{~d} z) \lesssim r^{\theta^{\prime}-\alpha}
$$


Proof. Noting $\theta>\alpha$, one sees that

$$
\begin{aligned}
\int_{|z| \leqslant r}|z|^{\theta} v(\mathrm{~d} z) & \leqslant \sum_{k=0}^{\infty}\left(2^{-k} r\right)^{\theta-2} \int_{2^{-k-1} r<|z| \leqslant 2^{-k} r}|z|^{2} v(\mathrm{~d} z) \\
& \lesssim r^{\theta-\alpha} \sum_{k=0}^{\infty} 2^{k(\alpha-\theta)} \lesssim r^{\theta-\alpha} .
\end{aligned}
$$

Similarly,

$$
\begin{aligned}
\int_{r<|z| \leqslant 1}|z|^{\theta^{\prime}} v(\mathrm{~d} z) & \leqslant \int_{r<|z| \leqslant \rho}|z|^{\theta^{\prime}} v(\mathrm{~d} z)+\rho^{\theta^{\prime}} \int_{\rho<|z| \leqslant 1} v(\mathrm{~d} z) \\
& \lesssim \sum_{k=0}^{\log _{2}(\rho / r)}\left(2^{-k} \rho\right)^{\theta^{\prime}-2} \int_{2^{-k-1} \rho<|z| \leqslant 2^{-k} \rho}|z|^{2} v(\mathrm{~d} z)+1 \lesssim r^{\theta^{\prime}-\alpha}
\end{aligned}
$$

We also need the following lemma (see [Tri92, Theorem 2.4.7]) in order to localize the resolvent equation.

Lemma 3.6 (localization principle). Let $\zeta_{k} \in C_{c}^{\infty}, k=1,2, \cdots$. Assume for any multi-index $\alpha$, $\sup _{x \in \mathbb{R}^{d}} \sum_{k}\left|\partial^{\alpha} \zeta_{k}(x)\right| \leqslant C_{\alpha}<\infty$. Then there is a constant $C$ such that

$$
\sum_{k}\left\|u \zeta_{k}\right\|_{W_{p}^{s}}^{p} \leqslant C\|u\|_{W_{p}^{s}}^{p}
$$

Moreover, if $\sup _{x \in \mathbb{R}^{d}} \sum_{k}\left|\zeta_{k}(x)\right|^{p} \geqslant c>0$, then we have

$$
\|u\|_{W_{p}^{s}}^{p} \asymp \sum_{k}\left\|u \zeta_{k}\right\|_{W_{p}^{s}}^{p} .
$$

The following lemma is taken from [MP92, Lemma 3.5].

Lemma 3.7. Suppose $s \in(0,2), p>\max \left\{1, \frac{d}{s}\right\}$, then

$$
\left\|\sup _{y \neq 0} \frac{\left|f(\cdot+y)-f(\cdot)-\nabla f(\cdot) \cdot y^{(s)}\right|}{|y|^{s}}\right\|_{p} \leqslant C\|f\|_{H_{p}^{s}},
$$

where $y^{(s)}=y \mathbf{1}_{s \in(1,2)}+y \mathbf{1}_{s=1} \mathbf{1}_{B_{1}}(y)$.

Theorem 3.8. Let $\alpha \in(0,2),(1-\alpha)^{+}<\gamma<\beta<1$ and $p>\frac{d}{\alpha \wedge 1} \vee \frac{d}{\alpha+\gamma-1}$. Assume $v$ is supported on $B_{R}$ satisfying conditions $\left(\mathrm{A}_{1}\right), \sigma$ satisfies $\left(\mathrm{A}_{2}\right)$ and $b \in C_{b}^{\beta}$. Then there is a constant $\lambda_{0}$ such that for any $\lambda \geqslant \lambda_{0}$ and $f \in \mathscr{W}_{p}^{\gamma}$, equation (3.1) admits a unique solution in $\mathscr{W}_{p}^{\alpha+\gamma}$. Moreover,

$$
\lambda\|u\|_{\mathscr{W}_{p}^{\gamma}}+\|u\|_{\mathscr{W}_{p}^{\alpha+\gamma}} \leqslant C\|f\|_{\mathscr{W}_{p}^{\gamma}}
$$

where $\lambda_{0}, C$ depend only on $d, p, \alpha, \beta, \gamma, R, c_{0}, \rho, \Lambda$ and $\|b\|_{C_{b}^{\beta}}$. 
When $\sigma$ is independent of $x$, then we do not need to assume $v$ is compactly supported (see Remark 1.2). However, if $\sigma$ does depend on $x$ and the Lévy measure $v$ is not necessarily absolutely continuous w.r.t. the Lebesgue measure, it seems not feasible to show that for any $f \in W_{p}^{\gamma}$,

$$
x \mapsto \int_{|z| \geqslant R} f(x+\sigma(x) z) v(\mathrm{~d} z) \in W_{p}^{\gamma}
$$

which is very essential if one wants to use the perturbation argument. So in this case, we need to assume supp $v \subseteq B_{R}$ with some constant $R \geqslant 1$. Thus, $\mathscr{L} f(x)$ can be rewritten as

$$
\mathscr{L} f(x)=\int_{B_{R}}\left(f(x+\sigma(x) z)-f(x)-\nabla f(x) \cdot \sigma(x) z \mathbf{1}_{B_{1}}(z)\right) v(\mathrm{~d} z) .
$$

In order to prove Theorem 3.8, we need a commutator estimate under the following assumption: there are $\varepsilon \in(0,1)$ and $\Lambda \geqslant 1$ such that

$$
\begin{array}{r}
|\sigma(x)-\sigma(y)| \leqslant \Lambda|x-y|, \sigma(x)=\sigma(0), \quad \forall|x| \geqslant \varepsilon \\
\Lambda^{-1}|\xi|^{2} \leqslant|\sigma(0) \xi|^{2} \leqslant \Lambda|\xi|^{2}, \forall \xi \in \mathbb{R}^{d} .
\end{array}
$$

If $\operatorname{supp} v \subseteq B_{R}$, we denote

$$
L_{\sigma}^{\alpha} f(x):=\int_{B_{R}}\left(f(x+\sigma(x) z)-f(x)-\nabla f(x) \cdot \sigma(x) z \mathbf{1}_{\alpha \in[1,2)}\right) v(\mathrm{~d} z) .
$$

Lemma 3.9. Suppose $v$ is supported on $B_{R}$ satisfying $\left(\mathrm{A}_{1}\right)$ and $\sigma$ satisfies (3.14). Then for any $s \in(0,1), p>1$, we have

$$
\left\|\left[\Delta^{s / 2}, L_{\sigma}^{\alpha}\right] u\right\|_{p} \leqslant C \varepsilon^{1-s+\frac{d}{p}}\left\{\begin{array}{lr}
\|u\|_{C_{b}^{1}}, & \alpha \in(0,1) \\
\|u\|_{C_{b}^{\alpha+\delta}}, & \alpha \in[1,2), \delta>0
\end{array}\right.
$$

where $\left[\Delta^{s / 2}, L_{\sigma}^{\alpha}\right] u:=\Delta^{s / 2} L_{\sigma}^{\alpha} u-L_{\sigma}^{\alpha} \Delta^{s / 2} u$, and the constant $C>0$ is independent of $\varepsilon$.

The reader can find the proof of above lemma in [CZZ21].

Lemma 3.10. Suppose $v$ is supported on $B_{R}$ satisfying $\left(\mathrm{A}_{1}\right)$ and $\sigma$ satisfies (3.14). Then for any $\gamma \in(0,1), p \in\left(\frac{d}{\alpha \wedge 1}, \infty\right)$,we have

$$
\left\|\left(L_{\sigma}^{\alpha}-L_{\sigma(0)}^{\alpha}\right) f\right\|_{W_{p}^{\gamma}} \leqslant c_{\varepsilon}\|f\|_{W_{p}^{\alpha+\gamma}}
$$

where $c_{\varepsilon} \rightarrow 0$ as $\varepsilon \rightarrow 0$.

Proof. We only prove the estimate for $\alpha \in(0,1)$. The case $\alpha \in[1,2)$ is similar. Without loss of generality, we can assume $R=1$. Define

$$
\mathscr{T}_{\sigma} f:=L_{\sigma}^{\alpha} f-L_{\sigma(0)}^{\alpha} f=\int_{|z| \leqslant 1}(f(x+\sigma(x) z)-f(x+\sigma(0) z)) v(\mathrm{~d} z) .
$$

For $\theta \in(d / p, 1], \delta>0$ and $y_{0} \in \mathbb{R}^{d}$, by (3.12), we have

$$
\left\|\sup _{\left|y-y_{0}\right| \leqslant \delta}\left|f(\cdot+y)-f\left(\cdot+y_{0}\right)\right|\right\|_{p} \lesssim \delta^{\theta}\|f\|_{H_{p}^{\theta}}
$$


which implies that

$$
\begin{aligned}
& \|f(\cdot+\sigma(\cdot) z)-f(\cdot+\sigma(0) z)\|_{p} \\
& \leqslant\left\|\operatorname { s u p } _ { | y - \sigma ( 0 ) z | \leqslant \Lambda \varepsilon | z | } \left|f(\cdot+y)-f(\cdot+\sigma(0) z)\left\|_{p} \lesssim \varepsilon^{\theta}|z|^{\theta}\right\| f \|_{H_{p}^{\theta}}\right.\right.
\end{aligned}
$$

Thus, due to $p>\frac{d}{\alpha}$, we can choose $\frac{d}{p}<\theta^{\prime}<\alpha<\theta \leqslant 1$ such that

$$
\left\|\mathscr{T}_{\sigma} f\right\|_{p} \lesssim \int_{|z| \leqslant 1}\left(\varepsilon^{\theta}|z|^{\theta}\|f\|_{H_{p}^{\theta}}\right) \wedge\left(\varepsilon^{\theta^{\prime}}|z|^{\theta^{\prime}}\|f\|_{H_{p}^{\theta^{\prime}}}\right) v(\mathrm{~d} z),
$$

which together with Bernstein's inequality implies

$$
\begin{aligned}
\left\|\mathscr{T}_{\sigma} \Delta_{j} f\right\|_{p} & \lesssim \int_{|z| \leqslant 1}\left(\varepsilon^{\theta}|z|^{\theta}\left\|\Delta_{j} f\right\|_{H_{p}^{\theta}}\right) \wedge\left(\varepsilon^{\theta^{\prime}}|z|^{\theta^{\prime}}\left\|\Delta_{j} f\right\|_{H_{p}^{\theta^{\prime}}}\right) v(\mathrm{~d} z) \\
& \lesssim \int_{|z| \leqslant 1}\left(\varepsilon^{\theta}|z|^{\theta} 2^{\theta j}\right) \wedge\left(\varepsilon^{\theta^{\prime}}|z|^{\theta^{\prime}} 2^{\theta^{\prime} j}\right) v(\mathrm{~d} z)\left\|\Delta_{j} f\right\|_{p} .
\end{aligned}
$$

By (3.9) and (3.10)

$$
\begin{aligned}
& \int_{|z| \leqslant 1}\left(\varepsilon^{\theta}|z|^{\theta} 2^{\theta j}\right) \wedge\left(\varepsilon^{\theta^{\prime}}|z|^{\theta^{\prime}} 2^{\theta^{\prime} j}\right) v(\mathrm{~d} z) \\
= & \varepsilon^{\theta} 2^{\theta j} \int_{|z| \leqslant 2^{-j}}|z|^{\theta} v(\mathrm{~d} z)+\varepsilon^{\theta^{\prime}} 2^{\theta^{\prime} j} \int_{2^{-j}<|z| \leqslant 1}|z|^{\theta^{\prime}} v(\mathrm{~d} z) \lesssim \varepsilon^{\theta^{\prime}} 2^{\alpha j} .
\end{aligned}
$$

Substituting the above estimate into (3.16), we obtain

$$
\left\|\mathscr{T}_{\sigma} \Delta_{j} f\right\|_{p} \lesssim \varepsilon^{\theta^{\prime}} 2^{\alpha j}\left\|\Delta_{j} f\right\|_{p} .
$$

Note that

$$
\left\|\Delta_{i} \mathscr{T}_{\sigma} f\right\|_{p} \leqslant \sum_{j>i}\left\|\Delta_{i} \mathscr{T}_{\sigma} \Delta_{j} f\right\|_{p}+\sum_{j \leqslant i}\left\|\Delta_{i} \mathscr{T}_{\sigma} \Delta_{j} f\right\|_{p}=: \mathscr{J}_{i}^{(1)}+\mathscr{J}_{i}^{(2)}
$$

For $\mathscr{J}_{i}^{(1)}$, by (3.17), we have

$$
\mathscr{J}_{i}^{(1)} \lesssim \varepsilon^{\theta^{\prime}} \sum_{j>i} 2^{\alpha j}\left\|\Delta_{j} f\right\|_{p} \lesssim \varepsilon^{\theta^{\prime}}\|f\|_{W_{p}^{\alpha+\gamma}} \sum_{j>i} 2^{-\gamma j} c_{j},
$$

where $c_{j}:=2^{(\alpha+\gamma) j}\left\|\Delta_{j} f\right\|_{p} /\|f\|_{W_{p}^{\alpha+\gamma}}$. Hence,

$$
\begin{aligned}
2^{\gamma i} \mathscr{J}_{i}^{(1)} & \lesssim \varepsilon^{\theta^{\prime}}\|f\|_{W_{p}^{\alpha+\gamma}} \sum_{j \in \mathbb{Z}} \mathbf{1}_{\{(i-j)<0\}} 2^{\gamma(i-j)} \cdot \mathbf{1}_{\{j \geqslant-1\}} c_{j} \\
& =\varepsilon^{\theta^{\prime}}\|f\|_{W_{p}^{\alpha+\gamma}}(a * b)_{i},
\end{aligned}
$$

where $a_{k}=\mathbf{1}_{\{k \geqslant-1\}} c_{k}$ and $b_{k}=\mathbf{1}_{\{k<0\}} 2^{\gamma k}(\forall k \in \mathbb{Z})$. This yields

$$
\begin{gathered}
\left\|2^{\gamma i} \mathscr{J}_{i}^{(1)}\right\|_{\ell^{p}} \lesssim \varepsilon^{\theta^{\prime}}\|f\|_{W_{p}^{\alpha+\gamma}}\|a\|_{\ell^{p}}\|b\|_{\ell^{1}} \\
\lesssim \varepsilon^{\theta^{\prime}}\|f\|_{W_{p}^{\alpha+\gamma}}\left(\sum_{j \geqslant-1} c_{j}^{p}\right)^{1 / p} \lesssim \varepsilon^{\theta^{\prime}}\|f\|_{W_{p}^{\alpha+\gamma}}
\end{gathered}
$$


For $\mathscr{J}_{i}^{(2)}$, if $i=-1$, then

$$
\mathscr{J}_{-1}^{(2)}=\left\|\Delta_{-1} \mathscr{T}_{\sigma} \Delta_{-1} f\right\|_{p} \lesssim \varepsilon^{\alpha}\left\|\Delta_{-1} f\right\|_{H_{p}^{\alpha}} \lesssim \varepsilon^{\alpha}\|f\|_{W_{p}^{\alpha+\gamma} c_{-1}}
$$

If $i \geqslant 0$, choose $s \in(\gamma \vee(1-\alpha+d / p), 1)$ in Lemma 3.9. By Bernstein's inequality and Lemma 3.9, we have

$$
\begin{aligned}
& \mathscr{J}_{i}^{(2)}=\sum_{-1 \leqslant j \leqslant i}\left\|\Delta_{i} \Delta^{-s / 2} \Delta^{s / 2} \mathscr{T}_{\sigma} \Delta_{j} f\right\|_{p} \stackrel{(2.5)}{\lesssim} 2^{-s i} \sum_{-1 \leqslant j \leqslant i}\left\|\Delta^{s / 2} \mathscr{T}_{\sigma} \Delta_{j} f\right\|_{p} \\
& \leqslant 2^{-s i} \sum_{-1 \leqslant j \leqslant i}\left(\left\|\left[\Delta^{s / 2}, \mathscr{T}_{\sigma}\right] \Delta_{j} f\right\|_{p}+\left\|\mathscr{T}_{\sigma} \Delta^{s / 2} \Delta_{j} f\right\|_{p}\right) \\
& =2^{-s i} \sum_{-1 \leqslant j \leqslant i}\left(\left\|\left[\Delta^{s / 2}, L_{\sigma}^{\alpha}\right] \Delta_{j} f\right\|_{p}+\left\|\mathscr{T}_{\sigma} \Delta_{j} \Delta^{s / 2} f\right\|_{p}\right) \\
& \stackrel{(3.15),(3.17)}{\lesssim} 2^{-s i} c_{\varepsilon} \sum_{-1 \leqslant j \leqslant i}\left(\left\|\Delta_{j} f\right\|_{C_{b}^{1}}+\left\|\Delta^{s / 2} \Delta_{j} f\right\|_{H_{p}^{\alpha}}\right) \\
& \stackrel{(2.4)}{\lesssim} 2^{-s i} c_{\varepsilon} \sum_{-1 \leqslant j \leqslant i}\left(2^{(1+d / p) j}\left\|\Delta_{j} f\right\|_{p}+2^{(s+\alpha) j}\left\|\Delta_{j} f\right\|_{p}\right) \\
& \lesssim c_{\varepsilon} \sum_{-1 \leqslant j \leqslant i} 2^{(\alpha+s) j}\left\|\Delta_{j} f\right\|_{p} \\
& \lesssim c_{\varepsilon}\|f\|_{W_{p}^{\alpha+\gamma}} 2^{-s i} \sum_{-1 \leqslant j \leqslant i} 2^{(s-\gamma) j} c_{j}
\end{aligned}
$$

Denoting $a_{k}=\mathbf{1}_{\{k \geqslant-1\}} c_{k}, d_{k}=\mathbf{1}_{\{k \geqslant 0\}} 2^{(\gamma-s) k}$, then

$$
2^{\gamma i} \mathscr{J}_{i}^{(2)} \leqslant c_{\varepsilon}\|f\|_{W_{p}^{\alpha+\gamma}} \sum_{-1 \leqslant j \leqslant i} 2^{(\gamma-s)(i-j)} c_{j} \leqslant c_{\varepsilon}\|f\|_{W_{p}^{\alpha+\gamma}}(d * a)_{i} .
$$

Thus,

$$
\left\|2^{\gamma i} \mathscr{J}_{i}^{(2)}\right\|_{\ell^{p}} \lesssim c_{\mathcal{\varepsilon}}\|f\|_{W_{p}^{\alpha+\gamma}}\|d\|_{\ell^{1}}\|a\|_{\ell^{p}} \lesssim c_{\mathcal{\varepsilon}}\|f\|_{W_{p}^{\alpha+\gamma}}
$$

By (3.18) and (3.19), we get

$$
\begin{aligned}
\left\|\mathscr{T}_{\sigma} f\right\|_{W_{p}^{\gamma}} & \asymp\left\|2^{\gamma i}\right\| \Delta_{i} \mathscr{T}_{\sigma} f\left\|_{p}\right\|_{\ell^{p}} \leqslant\left\|2^{\gamma i} \mathscr{J}_{i}^{(1)}\right\|_{\ell^{p}}+\left\|2^{\gamma i} \mathscr{J}_{i}^{(2)}\right\|_{\ell^{p}} \\
& \lesssim c_{\mathcal{E}}\|f\|_{W_{p}^{\alpha+\gamma}}
\end{aligned}
$$

This yields our desired result.

Now we are on the position to prove Theorem 3.8.

Proof of Theorem 3.8. Like before, we only give the a prior estimate here. Assume $\left\{\zeta_{k}\right\}_{k \in \mathbb{N}}$ is a standard partition of unity and for each $k$, the support of $\zeta_{k}$ lies in a ball $B_{\varepsilon / 8}\left(y_{k}\right)$, and $\varepsilon>0$ will be determined later. Also for any $k$, we take functions $\eta_{k}, \xi_{k} \in C_{c}^{\infty}\left(\mathbb{R}^{d}\right)$ such that $\eta_{k}=1$ on $B_{\varepsilon / 4}\left(y_{k}\right)$, $\eta_{k}=0$ outside $B_{\varepsilon / 2}\left(y_{k}\right)$, and $0 \leqslant \eta_{k} \leqslant 1 ; \xi_{k}=1$ on $B_{\varepsilon / 2}\left(y_{k}\right), \xi_{k}=0$ outside $B_{\varepsilon}\left(y_{k}\right)$, and $0 \leqslant \xi_{k} \leqslant 1$. Define $\sigma_{k}(x)=\xi_{k}(x) \sigma(x)+\left(1-\xi_{k}(x)\right) \sigma\left(y_{k}\right)$,

$$
\mathscr{L}_{k} f(x):=\int_{B_{R}}\left(f\left(x+\sigma_{k}(x) z\right)-f(x)-\nabla f(x) \sigma_{k}(x) z \mathbf{1}_{B_{1}}(z)\right) v(\mathrm{~d} z) .
$$




$$
L_{k} f(x):=\int_{B_{R}}\left(f\left(x+\sigma\left(y_{k}\right) z\right)-f(x)-\nabla f(x) \sigma\left(y_{k}\right) z \mathbf{1}_{B_{1}}(z)\right) v(\mathrm{~d} z) .
$$

Multiplying $\zeta_{k}$ on both side of (3.1), we get

$$
\lambda\left(u \zeta_{k}\right)-L_{k}\left(u \zeta_{k}\right)-b \cdot \nabla\left(u \zeta_{k}\right)=f \zeta_{k}+\zeta_{k}(b \cdot \nabla u)-b \cdot \nabla\left(u \zeta_{k}\right)+\zeta_{k}(\mathscr{L} u)-L_{k}\left(\zeta_{k} u\right) .
$$

Lemma 3.4 yields,

$$
\lambda\left\|u \zeta_{k}\right\|_{W_{p}^{\gamma}}+\left\|u \zeta_{k}\right\|_{W_{p}^{\alpha+\gamma}} \lesssim\left(\left\|f \zeta_{k}\right\|_{W_{p}^{\gamma}}+\left\|u b \cdot \nabla \zeta_{k}\right\|_{W_{p}^{\gamma}}+\left\|\zeta_{k}(\mathscr{L} u)-L_{k}\left(\zeta_{k} u\right)\right\|_{W_{p}^{\gamma}}\right) .
$$

So by Lemma 3.6, we have,

$$
\begin{aligned}
\lambda^{p}\|u\|_{W_{p}^{\gamma}}^{p}+\|u\|_{W_{p}^{\alpha+\gamma}}^{p} & \lesssim \sum_{k} \lambda^{p}\left\|u \zeta_{k}\right\|_{W_{p}^{\gamma}}^{p}+\left\|u \zeta_{k}\right\|_{W_{p}^{\alpha+\gamma}}^{p} \\
& \lesssim \sum_{k}\left(\left\|f \zeta_{k}\right\|_{W_{p}^{\gamma}}^{p}+\left\|u b \cdot \nabla \zeta_{k}\right\|_{W_{p}^{\gamma}}^{p}+\left\|\zeta_{k}(\mathscr{L} u)-L_{k}\left(\zeta_{k} u\right)\right\|_{W_{p}^{\gamma}}^{p}\right)
\end{aligned}
$$

Again by Lemma 3.6,

$$
\sum_{k}\left\|f \zeta_{k}\right\|_{W_{p}^{\gamma}}^{p} \asymp\|f\|_{W_{p}^{\gamma}}^{p}, \sum_{k}\left\|u b \cdot \nabla \zeta_{k}\right\|_{W_{p}^{\gamma}}^{p} \lesssim\|u b\|_{W_{p}^{\gamma}}^{p} \lesssim\|u\|_{W_{p}^{\gamma}}^{p}
$$

the last inequality above is due to the following fact:

$$
\begin{aligned}
&\|u b\|_{W_{p}^{\gamma}}^{p} \lesssim\|u b\|_{p}^{p}+\iint_{\mathbb{R}^{d} \times \mathbb{R}^{d}} \frac{|u b(x)-u b(y)|^{p}}{|x-y|^{d+\gamma p}} \mathrm{~d} x \mathrm{~d} y \\
& \leqslant\|u\|_{p}^{p}\|b\|_{L^{\infty}}^{p} \mathrm{~d} x \mathrm{~d} y+\iint_{\mathbb{R}^{d} \times \mathbb{R}^{d}} \frac{|u(x)-u(y)|^{p}|b(x)|^{p}}{|x-y|^{d+\gamma p}} \\
&+\iint_{\mathbb{R}^{d} \times \mathbb{R}^{d}} \frac{|u(y)|^{p}|b(x)-b(y)|^{p}}{|x-y|^{d+\gamma p}} \mathrm{~d} x \mathrm{~d} y \\
& \lesssim\|u\|_{p}^{p}\|b\|_{L^{\infty}}^{p}+\|b\|_{L^{\infty}}^{p}[u]_{\gamma, p}^{p}+\int_{\mathbb{R}^{d}}|u(y)|^{p} \mathrm{~d} y \int_{\mathbb{R}^{d}} \frac{[b]_{\beta}^{p}|x-y|^{\beta p} \wedge\|b\|_{L^{\infty}}^{p}}{|x-y|^{d+\gamma p}} \mathrm{~d} x \\
& \lesssim\|u\|_{W_{p}^{\gamma}}^{p}\|b\|_{C_{b}^{\beta}}^{p} .
\end{aligned}
$$

Next we estimate the third term in the last line of (3.21). We only give the proof for $\alpha<1$ below, since the proof for $\alpha \geqslant 1$ is almost the same. Rewrite

$$
\begin{aligned}
& \zeta_{k}(x)(\mathscr{L} u)(x)-L_{k}\left(\zeta_{k} u\right)(x) \\
= & {\left[\mathscr{L}\left(u \zeta_{k}\right)(x)-L_{k}\left(u \zeta_{k}\right)(x)\right] \eta_{k}(x)+\left[\mathscr{L}\left(u \zeta_{k}\right)(x)-L_{k}\left(u \zeta_{k}\right)(x)\right]\left(1-\eta_{k}(x)\right) } \\
& -\left\{u(x) \mathscr{L} \zeta_{k}(x)+\int_{B_{R}}[u(x+\sigma(x) z)-u(x)] \cdot\left[\zeta_{k}(x+\sigma(x) z)-\zeta_{k}(x)\right] v(\mathrm{~d} z)\right\} \\
= & : I_{k}^{(1)}(x)+I_{k}^{(2)}(x)-I_{k}^{(3)}(x) .
\end{aligned}
$$

For $I_{k}^{(1)}$, notice $\sigma_{k}(x)=\sigma(x)$ when $x$ is in the support of $\eta_{k}$, so we have

$$
\begin{aligned}
I_{k}^{(1)}(x) & =\left[\mathscr{L}_{k}\left(u \zeta_{k}\right)(x)-L_{k}\left(u \zeta_{k}\right)(x)\right] \eta_{k}(x) \\
& =\left[L_{\sigma_{k}}^{\alpha}\left(u \zeta_{k}\right)(x)-L_{\sigma\left(y_{k}\right)}^{\alpha}\left(u \zeta_{k}\right)(x)\right] \eta_{k}(x) .
\end{aligned}
$$


By Lemma 3.10, we have

$$
\left\|I_{k}^{(1)}\right\|_{W_{p}^{\gamma}} \leqslant c_{\varepsilon}\left\|u \zeta_{k}\right\|_{W_{p}^{\alpha+\gamma}}\left(c_{\varepsilon} \rightarrow 0 \text { as } \varepsilon \rightarrow 0\right) .
$$

For $I_{k}^{(2)}(x)$, since $1-\eta_{k}(x)=0$ if $\left|x-y_{k}\right| \leqslant \frac{\varepsilon}{4}$ and $u \zeta_{k}(x)=0$ if $\left|x-y_{k}\right|>\frac{\varepsilon}{8}$, we have

$$
I_{k}^{(2)}(x)=\int_{\frac{\varepsilon}{8 \Lambda} \leqslant|z|<R}\left[u \zeta_{k}(x+\sigma(x) z)-u \zeta_{k}\left(x+\sigma\left(y_{k}\right) z\right)\right]\left(1-\eta_{k}(x)\right) v(\mathrm{~d} z) .
$$

Choosing $s \in(d / p, 1 \wedge(\alpha+\gamma-1))$, by Minkowski inequality, Lemma 3.7 and interpolation theorem, we have

$$
\begin{aligned}
\left\|I_{k}^{(2)}\right\|_{p} & \leqslant \int_{\frac{\varepsilon}{8 \Lambda} \leqslant|z|<R}\left\|u \zeta_{k}(\cdot+\sigma(\cdot) z)-u \zeta_{k}\left(\cdot+\sigma\left(y_{k}\right) z\right)\right\|_{p} v(\mathrm{~d} z) \\
& \lesssim \int_{\frac{\varepsilon}{8 \Lambda} \leqslant|z|<R}\left\|\sup _{|y| \leqslant 2 \Lambda|z|}\left|u \zeta_{k}(\cdot+y)-u \zeta_{k}(\cdot)\right|\right\|_{p} v(\mathrm{~d} z) \\
& \stackrel{(3.12)}{\lesssim}\left\|u \zeta_{k}\right\|_{H_{p}^{s}} \int_{\frac{\varepsilon}{8 \Lambda} \leqslant|z|<R}|z|^{s} v(\mathrm{~d} z) \lesssim \varepsilon^{s-\alpha}\left\|u \zeta_{k}\right\|_{H_{p}^{s}} \\
& \leqslant \varepsilon\left\|u \zeta_{k}\right\|_{W_{p}^{\alpha+\gamma}+C_{\varepsilon}}\left\|u \zeta_{k}\right\|_{p} .
\end{aligned}
$$

Moreover, noticing that

$$
\begin{aligned}
\nabla I_{k}^{(2)}(x)= & \int_{\frac{\varepsilon}{8 \Lambda} \leqslant|z|<R}\left\{\left[\nabla\left(u \zeta_{k}\right)(x+\sigma(x) z)(\mathbb{I}+\nabla \sigma(x) z)-\nabla\left(u \zeta_{k}\right)\left(x+\sigma\left(y_{k}\right) z\right)\right]\right. \\
& \left.\left(1-\eta_{k}(x)\right)-\left[u \zeta_{k}(x+\sigma(x) z)-u \zeta_{k}\left(x+\sigma\left(y_{k}\right) z\right)\right] \nabla \eta_{k}(x)\right\} v(\mathrm{~d} z) \\
= & \int_{\frac{\varepsilon}{8 \Lambda} \leqslant|z|<R}\left\{\nabla\left(u \zeta_{k}\right)(x+\sigma(x) z) \cdot \nabla \sigma(x) z\left(1-\eta_{k}(x)\right)\right. \\
& +\left[\nabla\left(u \zeta_{k}\right)(x+\sigma(x) z)-\nabla\left(u \zeta_{k}\right)\left(x+\sigma\left(y_{k}\right) z\right)\right]\left(1-\eta_{k}(x)\right) \\
& \left.-\left[u \zeta_{k}(x+\sigma(x) z)-u \zeta_{k}\left(x+\sigma\left(y_{k}\right) z\right)\right] \nabla \eta_{k}(x)\right\} v(\mathrm{~d} z),
\end{aligned}
$$

like before, we choose $s \in(d / p, 1 \wedge(\alpha+\gamma-1))$, then

$$
\begin{aligned}
&\left\|\nabla I_{k}^{(2)}\right\|_{p} \lesssim \int_{\frac{\varepsilon}{8 \Lambda} \leqslant|z|<R}\left\{\left[\left\|\nabla\left(u \zeta_{k}\right)(\cdot+\sigma(\cdot) z)-\nabla\left(u \zeta_{k}\right)(\cdot)\right\|_{p}+\left\|\nabla\left(u \zeta_{k}\right)\right\|_{p}\right] \cdot\|\nabla \sigma\|_{\infty}|z|\right. \\
&+\left\|\nabla\left(u \zeta_{k}\right)(\cdot+\sigma(\cdot) z)-\nabla\left(u \zeta_{k}\right)\left(\cdot+\sigma\left(y_{k}\right) z\right)\right\|_{p} \\
&\left.+\left\|\nabla \eta_{k}\right\|_{\infty} \cdot\left\|u \zeta_{k}(\cdot+\sigma(\cdot) z)-u \zeta_{k}\left(\cdot+\sigma\left(y_{k}\right) z\right)\right\|_{p}\right\} v(\mathrm{~d} z) \\
& \stackrel{(3.12)}{\lesssim \varepsilon} \int_{\frac{\varepsilon}{8 \Lambda} \leqslant|z|<R}\left(|z|^{1+s}+|z|^{s}\right)\left\|\sup _{y \in \mathbb{R}^{d}} \frac{\left|\nabla\left(u \zeta_{k}\right)(\cdot+y)-\nabla\left(u \zeta_{k}\right)(\cdot)\right|}{|y|^{s}}\right\| \|_{p} v(\mathrm{~d} z) \\
&+\int_{\frac{\varepsilon}{8 \Lambda} \leqslant|z|<R}|z|\left\|\nabla\left(u \zeta_{k}\right)\right\|_{p} v(\mathrm{~d} z) \\
& \lesssim \varepsilon \varepsilon\left\|u \zeta_{k}\right\|_{H_{p}^{1+s}} \leqslant \varepsilon\left\|u \zeta_{k}\right\|_{W_{p}^{\alpha+\gamma}}+C_{\varepsilon}\left\|u \zeta_{k}\right\|_{p} .
\end{aligned}
$$


So

$$
\left\|I_{k}^{(2)}\right\|_{W_{p}^{\gamma}} \leqslant\left\|I_{k}^{(2)}\right\|_{W_{p}^{1}} \leqslant \varepsilon\left\|u \zeta_{k}\right\|_{W_{p}^{\alpha+\gamma}}+C_{\varepsilon}\left\|u \zeta_{k}\right\|_{p} .
$$

For $I_{k}^{(3)}(x)$, we have

$$
\sup _{x \in \mathbb{R}^{d}} \sum_{k}\left|\zeta_{k}(x+\sigma(x) z)-\zeta_{k}(x)\right|^{p} \lesssim|z|^{p}, \quad \forall|z|<R
$$

and

$$
\sup _{x \in \mathbb{R}^{d}} \sum_{k}\left|\left(\mathscr{L} \zeta_{k}\right)(x)\right|^{p} \lesssim 1
$$

Hence, for any $s \in(0, \alpha)$,

$$
\begin{aligned}
& \left(\sum_{k}\left\|I_{k}^{(3)}\right\|_{p}^{p}\right)^{1 / p} \leqslant\left(\sum_{k} \int_{\mathbb{R}^{d}}|u(x)|^{p}\left|\mathscr{L}_{k}(x)\right|^{p} \mathrm{~d} x\right)^{1 / p} \\
& +\int_{|z|<R}\left\{\int_{\mathbb{R}^{d}}|u(x+\sigma(x) z)-u(x)|^{p} \sum_{k}\left|\zeta_{k}(x+\sigma(x) z)-\zeta_{k}(x)\right|^{p} \mathrm{~d} x\right\}^{1 / p} v(\mathrm{~d} z) \\
\lesssim & \|u\|_{p}+\int_{|z|<R}\|u\|_{H_{p}^{s}}|z|^{s+1} v(\mathrm{~d} z) \leqslant \varepsilon\|u\|_{W_{p}^{\alpha+\gamma}+C_{\varepsilon}\|u\|_{p} .}
\end{aligned}
$$

Similarly, we have

$$
\left(\sum_{k}\left\|\nabla I_{k}^{(3)}\right\|_{p}^{p}\right)^{1 / p} \leqslant \varepsilon\|u\|_{W_{p}^{\alpha+\gamma}+C_{\varepsilon}\|u\|_{p}}
$$

So,

$$
\left(\sum_{k}\left\|I_{k}^{(3)}\right\|_{W_{p}^{\gamma}}^{p}\right)^{1 / p} \leqslant\left(\sum_{k}\left\|I_{k}^{(3)}\right\|_{W_{p}^{1}}^{p}\right)^{1 / p} \leqslant \varepsilon\|u\|_{W_{p}^{\alpha+\gamma}}+C_{\varepsilon}\|u\|_{p} .
$$

Now by Lemma 3.6, (3.21)-(3.25), and choosing $\varepsilon$ sufficiently small and $\lambda_{0}$ sufficiently large, we get

$$
\lambda\|u\|_{W_{p}^{\gamma}}+\|u\|_{W_{p}^{\alpha+\gamma}} \leqslant C\|f\|_{W_{p}^{\gamma}} .
$$

Now multiplying both sides of (3.1) by $\chi_{z}$, we have

$$
\lambda\left(u \chi_{z}\right)-\mathscr{L}\left(u \chi_{z}\right)-b \cdot \nabla\left(u \chi_{z}\right)=g_{z},
$$

where

$$
g_{z}:=f \chi_{z}+\chi_{z} \mathscr{L} u-\mathscr{L}\left(u \chi_{z}\right)-u b \cdot \nabla \chi_{z} .
$$

We omit the subscript $z$ below. By definition,

$$
[\chi \mathscr{L} u-\mathscr{L}(u \chi)](x)=\int_{B_{R}} u(x+\sigma(x) z)(\chi(x+\sigma(x) z)-\chi(x)) v(\mathrm{~d} z),
$$

so again by Lemma 3.7,

$$
\begin{aligned}
\|\chi \mathscr{L} u-\mathscr{L}(u \chi)\|_{p} & \leqslant\|u\|_{\infty}\left\|\int_{|z|<R}[\chi(\cdot+\sigma(\cdot) z)-\chi(\cdot)] v(\mathrm{~d} z)\right\|_{p} \\
& \leqslant C\|u\|_{\infty} .
\end{aligned}
$$


Noting that

$$
\begin{aligned}
\nabla[\chi \mathscr{L} u-\mathscr{L}(u \chi)](x)= & \int_{B_{R}} \nabla u(x+\sigma(x) z)(\mathbb{I}+\nabla \sigma(x) z)(\chi(x+\sigma(x) z)-\chi(x)) v(\mathrm{~d} z) \\
& +\int_{B_{R}} u(x+\sigma(x) z)(\nabla \chi(x+\sigma(x) z)(\mathbb{I}+\nabla \sigma(x) z)-\nabla \chi(x)) v(\mathrm{~d} z),
\end{aligned}
$$

we have

$$
\begin{aligned}
\|\nabla[\chi \mathscr{L} u-\mathscr{L}(u \chi)]\|_{p} \leqslant & C\|\nabla u\|_{\infty}\left\|\int_{B_{R}}[\chi(\cdot+\sigma(\cdot) z)-\chi(\cdot)] v(\mathrm{~d} z)\right\|_{p} \\
& +C\|u\|_{\infty}\left\|\int_{B_{R}}[\nabla \chi(\cdot+\sigma(\cdot) z)-\nabla \chi(\cdot)] v(\mathrm{~d} z)\right\|_{p} \\
& +C\|u\|_{\infty}\left\|\int_{B_{R}} \nabla \chi(\cdot+\sigma(\cdot) z) \cdot \nabla \sigma(x) z v(\mathrm{~d} z)\right\|_{p} \\
\leqslant & \|u\|_{C_{b}^{1}} .
\end{aligned}
$$

Thus,

$$
\begin{aligned}
\|g\|_{W_{p}^{\gamma}} & \leqslant\|f \chi\|_{W_{p}^{\gamma}}+C\|u\|_{C_{b}^{1}}+C\|b\|_{C_{b}^{\beta}}\|u \nabla \chi\|_{W_{p}^{\gamma}} \\
& \leqslant C\left(\|f\|_{\mathscr{W}_{p}^{\gamma}}+\|u\|_{C_{b}^{1}}+\|u\|_{\mathscr{W}_{p}^{\gamma}}\right) .
\end{aligned}
$$

By (3.26), we get

$$
\begin{aligned}
\|u\|_{\mathscr{W}_{p}^{\alpha+\gamma}}+\lambda\|u\|_{\mathscr{W}_{p}^{\gamma}} & =\sup _{z \in \mathbb{R}^{d}}\left(\left\|u \chi_{z}\right\|_{W_{p}^{\alpha+\gamma}}+\lambda\left\|u \chi_{z}\right\|_{W_{p}^{\gamma}}\right) \\
& \leqslant C \sup _{z \in \mathbb{R}^{d}}\left\|g_{z}\right\|_{W_{p}^{\gamma}} \leqslant\left(\|f\|_{\mathscr{W}_{p}^{\gamma}}+\|u\|_{C_{b}^{1}}+\|u\|_{\mathscr{W}_{p}^{\gamma}}\right) .
\end{aligned}
$$

By Lemma 2.6 and interpolation theorem, for any $\delta>0$ there is a constant $C_{\delta}>0$ such that

$$
\|u\|_{C_{b}^{1}} \leqslant \delta\|u\|_{\mathscr{W}^{\alpha+\gamma}}+C_{\delta}\|u\|_{\mathscr{W} \gamma}
$$

so we complete our proof by first choosing $\delta$ small and then choosing $\lambda_{0}$ sufficiently large.

Proof of Theorem 1.1. Our Theorem 1.1 is a consequence of Lemma 2.6 and Theorem 3.8.

\section{Proof of Theorem 1.3}

In this section, we prove our probabilistic results by using our analytic results proved in the previous section and Zvonkin's transform. Let $N(\mathrm{~d} t, \mathrm{~d} z)$ be the Poisson random measure associated with $Z$, that is,

$$
N((0, t] \times E)=\sum_{s \leqslant t} 1_{E}\left(\Delta Z_{s}\right),
$$

where $E$ is any compact set of $\mathbb{R}^{d} \backslash\{0\}$ and $\Delta Z_{s}:=Z_{s}-Z_{s-}$. The intensity measure of $N$ is denoted by $\mathrm{d} t v(\mathrm{~d} z)$. Let $\widetilde{N}(\mathrm{~d} t, \mathrm{~d} z)=N(\mathrm{~d} t, \mathrm{~d} z)-\mathrm{d} t v(\mathrm{~d} z)$, then

$$
Z_{t}=\int_{0}^{t} \int_{|z| \leqslant 1} z \widetilde{N}(\mathrm{~d} s, \mathrm{~d} z)+\int_{0}^{t} \int_{|z|>1} z N(\mathrm{~d} s, \mathrm{~d} z) .
$$


Thus, SDE (1.1) can be rewritten as

$$
X_{t}=X_{0}+\int_{0}^{t} b\left(X_{s}\right) \mathrm{d} s+\int_{0}^{t} \int_{|z| \leqslant 1} \sigma\left(X_{s-}\right) z \widetilde{N}(\mathrm{~d} s, \mathrm{~d} z)+\int_{0}^{t} \int_{|z|>1} \sigma\left(X_{s-}\right) z N(\mathrm{~d} s, \mathrm{~d} z) .
$$

Now we are on the point of giving the proof of our main results.

Proof of Theorem 1.3. (i). For the well-posedness, one can assume $v$ compactly supported on $B_{R}$ i.e. $\sup _{t \geqslant 0}\left|\Delta Z_{t}\right|<R$, otherwise, we can take $\tau_{0}:=0, \tau_{k}:=\inf \left\{t>\tau_{k-1}:\left|\Delta Z_{t}\right| \geqslant R\right\}$ for any $k \geqslant 1$, and solve the SDE step by step.

Now let $u$ be the solution of equation:

$$
\lambda u-\mathscr{L} u-b \cdot \nabla u=b, \quad \lambda \geqslant \lambda_{0}
$$

where

$$
\mathscr{L} u(x)=\int_{B_{R}}\left(u(x+z)-u(x)-\nabla u(x) \cdot \sigma(x) z \mathbf{1}_{B_{1}}(z)\right) v(\mathrm{~d} z)
$$

By Theorem 1.1 and interpolation theorem, for any $\mu \in(\alpha / 2, \alpha+\beta-1)$, we have $u \in C_{b}^{1+\mu}$ with $\|u\|_{C_{b}^{1+\mu}}=c(\lambda, \mu)$ and $c(\lambda, \mu) \rightarrow 0$ as $\lambda \rightarrow \infty$. Choose $\lambda$ sufficiently large so that $\|\nabla u\|_{\infty} \leqslant 1 / 2$, then $\phi: x \mapsto x+u(x)$ is a $C_{b}^{1+\mu}$-diffeomorphism. By (4.1) and a generalized version of Itô's formula(c.f. [Pri12]), we get

$$
\begin{aligned}
u\left(X_{t}\right)= & u\left(X_{0}\right)+\int_{0}^{t}[\mathscr{L} u+b \cdot \nabla u]\left(X_{s}\right) \mathrm{d} s \\
& +\int_{0}^{t} \int_{B_{R}}\left[u\left(X_{s-}+\sigma\left(X_{s-}\right) z\right)-u\left(X_{s-}\right)\right] \widetilde{N}(\mathrm{~d} s, \mathrm{~d} z) .
\end{aligned}
$$

Define $Y_{t}:=\phi\left(X_{t}\right)$, then

$$
\begin{aligned}
Y_{t} & =\phi\left(X_{t}\right)=\phi\left(X_{0}\right)+\int_{0}^{t} \lambda u\left(X_{s}\right) \mathrm{d} s \\
& +\int_{0}^{t} \int_{B_{R}}\left[\phi\left(X_{s-}+\sigma\left(X_{s-}\right) z\right)-\phi\left(X_{s-}\right)\right] \widetilde{N}(\mathrm{~d} s, \mathrm{~d} z) \\
& =Y_{0}+\int_{0}^{t} a\left(Y_{s}\right) \mathrm{d} s+\int_{0}^{t} \int_{B_{R}} g\left(Y_{s-}, z\right) \widetilde{N}(\mathrm{~d} s, \mathrm{~d} z),
\end{aligned}
$$

where

$$
\begin{gathered}
a(y):=\lambda u\left(\phi^{-1}(y)\right) \\
g(y, z):=\phi\left(\phi^{-1}(y)+\sigma\left(\phi^{-1}(y)\right) z\right)-y \\
=u\left(\phi^{-1}(y)+\sigma\left(\phi^{-1}(y)\right) z\right)-u\left(\phi^{-1}(y)\right)+\sigma\left(\phi^{-1}(y)\right) z .
\end{gathered}
$$

It has been showed in [Pri12] and [CSZ18] that in order to get the well-posedness of (4.1), we only need to show the well-posedness of (4.2). Elementary calculations yield,

$$
\nabla a(y)=\lambda \nabla u\left(\phi^{-1}(y)\right) \nabla \phi^{-1}(y)
$$




$$
\begin{aligned}
\nabla_{y} g(y, z)= & {\left[\nabla u\left(\phi^{-1}(y)+\sigma\left(\phi^{-1}(y)\right) z\right)-\nabla u\left(\phi^{-1}(y)\right)\right] \nabla \phi^{-1}(y) } \\
& +\nabla u\left(\phi^{-1}(y)+\sigma\left(\phi^{-1}(y)\right) z\right) \nabla \sigma\left(\phi^{-1}(y)\right) \nabla \phi^{-1}(y) z \\
& +\nabla \sigma\left(\phi^{-1}(y)\right) \nabla \phi^{-1}(y) z .
\end{aligned}
$$

Fix $\mu \in(\alpha / 2, \alpha+\beta-1)$. Noting that $u \in C_{b}^{1+\mu}$ with $\|u\|_{C_{b}^{1+\mu}}=c(\lambda, \mu)$, we have,

$$
\|a\|_{C_{b}^{1+\mu}}<\infty, \quad|g(y, z)| \leqslant C|z|
$$

and

$$
\begin{aligned}
\left\|\nabla_{y} g(\cdot, z)\right\|_{\infty} \leqslant & \|\nabla u\|_{C_{b}^{\mu}}\left\|\nabla \phi^{-1}\right\|_{\infty}\left(\|\sigma\|_{\infty} \cdot|z|\right)^{\mu} \\
& +\|\nabla u\|_{\infty}\|\nabla \sigma\|_{\infty}\left\|\nabla \phi^{-1}\right\|_{\infty}|z|+\|\nabla \sigma\|_{\infty}\left\|\nabla \phi^{-1}\right\|_{\infty}|z| \\
\leqslant & c(\lambda, \mu)(1-c(\lambda, \mu))^{-1}\left(\|\sigma\|_{\infty}^{\mu}|z|^{\mu}+\|\nabla \sigma\|_{\infty}|z|\right)+\|\nabla \sigma\|_{\infty}|z| .
\end{aligned}
$$

Thanks to the estimates (4.4) and (4.5), the proof for existence and uniqueness of solution to (4.2) becomes quite standard. Let

$$
Y_{t}^{0}=Y_{0} ; \quad Y_{t}^{n+1}=Y_{0}+\int_{0}^{t} a\left(Y_{s}^{n}\right) \mathrm{d} s+\int_{0}^{t} \int_{B_{R}} g\left(Y_{s-}^{n}, z\right) \widetilde{N}(\mathrm{~d} s, \mathrm{~d} z)
$$

then by Doob's inequality and (4.4), (4.5), we have

$$
\begin{aligned}
\mathbf{E} \sup _{0 \leqslant s \leqslant t}\left|Y_{s}^{n+1}-Y_{s}^{n}\right|^{2} \leqslant & C\|\nabla a\|_{\infty}^{2} \mathbf{E} \int_{0}^{t}\left|Y_{s}^{n}-Y_{s}^{n-1}\right|^{2} \mathrm{~d} s \\
& +C \mathbf{E} \int_{0}^{t} \int_{B_{R}}\left|g\left(Y_{s-}^{n}, z\right)-g\left(Y_{s-}^{n-1}, z\right)\right|^{2} v(\mathrm{~d} z) \mathrm{d} s \\
\leqslant & C\left(\|\nabla a\|_{\infty}^{2}+\int_{B_{R}}\left\|\nabla_{y} g(\cdot, z)\right\|_{\infty}^{2} v(\mathrm{~d} z)\right) \mathbf{E} \int_{0}^{t}\left|Y_{s}^{n}-Y_{s}^{n-1}\right|^{2} \mathrm{~d} s \\
& \leqslant C\left[1+\int_{B_{R}}\left(|z|^{2 \mu}+|z|^{2}\right) v(\mathrm{~d} z)\right] \mathbf{E} \int_{0}^{t}\left|Y_{s}^{n}-Y_{s}^{n-1}\right|^{2} \mathrm{~d} s \\
& \leqslant C \mathbf{E} \int_{0}^{t}\left|Y_{s}^{n}-Y_{s}^{n-1}\right|^{2} \mathrm{~d} s \leqslant C t \mathbf{E} \sup _{0 \leqslant s \leqslant t}\left|Y_{s}^{n}-Y_{s}^{n-1}\right|^{2} .
\end{aligned}
$$

Choosing $T$ sufficiently small such that $C T \leqslant \frac{1}{2}$, we get

$$
\lim _{n, m \rightarrow \infty} \mathbf{E} \sup _{0 \leqslant t \leqslant T}\left|Y_{t}^{n}-Y_{t}^{m}\right|^{2}=0
$$

Note that all the estimates above do not depends on the initial data $Y_{0}$, so we obtain that (4.6) holds for any $T>0$. The limit point $Y$ of $\left\{Y^{n}\right\}_{n}$ is a strong solution to (4.2). The uniqueness for (4.2) can be obtained by using Gronwall's inequality and similar estimates as above.

Next we show that for each $t, X_{t}$ is Malliavin differentiable. Noting that $\nabla\left(\phi^{-1}\right)(y)=(\nabla \phi)^{-1}$ 。 $\phi^{-1}(y)$ and $(\nabla \phi)^{-1}=\operatorname{adj}(\nabla \phi)(\operatorname{det} \nabla \phi)^{-1} \in C_{b}^{\mu}(\operatorname{adj}(\nabla \phi)$ is the adjugate matrix of $\nabla \phi)$, we get $\phi^{-1} \in C_{b}^{1+\mu}$. Since $X_{t}=\phi^{-1}\left(Y_{t}\right)$, by Theorem 12.8 of [DNØP09], we only need to show that $Y_{t}$ is 
Malliavin differentiable. By the closability of Malliavin derivative (Lemma 2.2), the desired result can be derived from following estimate:

$$
\sup _{n \in \mathbb{N} ; t \in[0, T]}\left\|D_{r, z} Y_{t}^{n}\right\|_{L^{2}(\lambda \times v \times \mathbf{P})}<\infty .
$$

Assume $Y_{t}^{n}$ above is Malliavin differentiable for each $t$ and $\sup _{t \in[0, T]}\left\|D_{r, z} Y_{t}^{n}\right\|_{L^{2}(\lambda \times v \times \mathbf{P})}<\infty$. By (4.4), we have

$$
a\left(Y_{s}^{n}\right) \in L^{2}(\mathbf{P}), \quad a\left(Y_{s}^{n}+D_{r, z} Y_{s}^{n}\right)-a\left(Y_{s}^{n}\right) \in L^{2}(\lambda \times v \times \mathbf{P}) .
$$

Thanks to Theorem 12.8 of [DNØP09], we obtain $a\left(Y_{s}^{n}\right) \in \mathbb{D}_{2}^{1}$ and

$$
D_{r, z} a\left(Y_{s}^{n}\right)=a\left(Y_{s}^{n}+D_{r, z} Y_{s}^{n}\right)-a\left(Y_{s}^{n}\right) \text {. }
$$

Similarly, by (4.4),

$$
\mathbf{E} \int_{0}^{T} \int_{\mathbb{R}^{d}}\left|g\left(Y_{s-}, \eta\right)\right|^{2} v(\mathrm{~d} \eta) \mathrm{d} s<\infty,
$$

and by (4.5) and Itô's isometry, we can see that for any $t \in[0, T]$,

$$
\begin{aligned}
& \mathbf{E}\left[\int_{0}^{t} \int_{\mathbb{R}^{d}}\left(\int_{0}^{t} \int_{\mathbb{R}^{d}} D_{r, z} g\left(Y_{s-}^{n}, \eta\right) \widetilde{N}(\mathrm{~d} s, \mathrm{~d} \eta)\right)^{2} v(\mathrm{~d} z) \mathrm{d} r\right] \\
&= \mathbf{E}\left[\int_{0}^{t} \int_{\mathbb{R}^{d}}\left(\int_{0}^{t} \int_{\mathbb{R}^{d}}\left[g\left(Y_{s}^{n}+D_{r, z} Y_{s-}^{n}, \eta\right)-g\left(Y_{s-}^{n}, \eta\right)\right] \widetilde{N}(\mathrm{~d} s, \mathrm{~d} \eta)\right)^{2} v(\mathrm{~d} z) \mathrm{d} r\right] \\
& \stackrel{(4.5)}{\leqslant} C \mathbf{E}\left[\int_{0}^{t} \int_{B_{R}}\left[\int_{0}^{t} \int_{B_{R}}\left\|\nabla_{y} g(\cdot, \eta)\right\|_{\infty}^{2}|\eta|^{2 \mu}\left|D_{r, z} Y_{s-}^{n}\right|^{2} v(\mathrm{~d} \eta) \mathrm{d} s\right] v(\mathrm{~d} z) \mathrm{d} r\right] \\
& \leqslant C \mathbf{E} \int_{0}^{T} \int_{B_{R}}\left[\int_{0}^{T}\left|D_{r, z} Y_{s-}^{n}\right|^{2} \mathrm{~d} s\right] v(\mathrm{~d} z) \mathrm{d} r \leqslant C \sup _{t \in[0, T]}\left\|D_{r, z} Y_{t}^{n}\right\|_{L^{2}(\lambda \times v \times \mathbf{P})}<\infty .
\end{aligned}
$$

Thanks to Theorem 12.15 of [DNØP09], we obtain

$$
\begin{aligned}
& D_{r, z} \int_{0}^{t} \int_{B_{R}} g\left(Y_{s-}^{n}, \eta\right) \widetilde{N}(\mathrm{~d} s, \mathrm{~d} \eta) \\
= & {\left[\int_{0}^{t} \int_{B_{R}}\left[g\left(Y_{s-}^{n}+D_{r, z} Y_{s-}^{n}, \eta\right)-g\left(Y_{s-}^{n}, \eta\right)\right] \widetilde{N}(\mathrm{~d} s, \mathrm{~d} \eta)+g\left(Y_{r-}^{n}, z\right)\right] \mathbf{1}_{[0, t]}(r) . }
\end{aligned}
$$

So we obtain $Y_{t}^{n+1} \in \mathbb{D}_{2}^{1}$ and for almost every $r \in[0, t]$,

$$
\begin{aligned}
D_{r, z} Y_{t}^{n+1}= & g\left(Y_{r-}^{n}, z\right)+\int_{r}^{t}\left[a\left(Y_{s}^{n}+D_{r, z} Y_{s}^{n}\right)-a\left(Y_{s}^{n}\right)\right] \mathrm{d} s \\
& +\int_{r}^{t} \int_{B_{R}}\left[g\left(Y_{s-}^{n}+D_{r, z} Y_{s-}^{n}, \eta\right)-g\left(Y_{s-}^{n}, \eta\right)\right] \widetilde{N}(\mathrm{~d} s, \mathrm{~d} \eta)
\end{aligned}
$$

For any $r \in[0, T]$, denote

$$
f_{r}^{n}=\mathbf{E}\left[\int_{B_{R}}\left[\sup _{r \leqslant t \leqslant T}\left|D_{r, z} Y_{t}^{n}\right|^{2}\right] v(\mathrm{~d} z)\right]
$$

Again by Doob's inequality,

$$
f_{r}^{n+1}=\mathbf{E}\left[\int_{B_{R}}\left[\sup _{r \leqslant t \leqslant T}\left|D_{r, z} Y_{t}^{n+1}\right|^{2}\right] v(\mathrm{~d} z)\right]
$$




$$
\begin{aligned}
\leqslant & C\left\{\int_{B_{R}}\|g(\cdot, z)\|_{\infty}^{2} v(\mathrm{~d} z)+\|\nabla a\|_{\infty}^{2} \mathbf{E} \int_{r}^{T} \int_{B_{R}}\left|D_{r, z} Y_{s-}^{n}\right|^{2} v(\mathrm{~d} z) \mathrm{d} s\right. \\
& \left.+\int_{B_{R}} \mathbf{E}\left[\int_{r}^{T} \int_{B_{R}}\left\|\nabla_{y} g(\cdot, \eta)\right\|_{\infty}^{2}\left|D_{r, z} Y_{s-}^{n}\right|^{2} v(\mathrm{~d} \eta) \mathrm{d} s\right] v(\mathrm{~d} z)\right\} \\
\leqslant & \left\{1+T \mathbf{E}\left[\int_{B_{R}}\left[\sup _{r \leqslant t \leqslant T}\left|D_{r, z} Y_{t-}^{n}\right|^{2}\right] v(\mathrm{~d} z)\right]\right. \\
& \left.+T \mathbf{E} \int_{B_{R}}\left[\sup _{r \leqslant t \leqslant T}\left|D_{r, z} Y_{t-}^{n}\right|^{2}\right] v(\mathrm{~d} z) \int_{B_{R}}|\eta|^{2 \mu} v(\mathrm{~d} \eta)\right\} \\
= & C+C T f_{r}^{n},
\end{aligned}
$$

where $C$ is independent with $n, r, T$. By choosing $T \leqslant T_{0}:=\frac{1}{2 C}$, then we have

$$
f_{r}^{n} \leqslant C+\frac{f_{r}^{n-1}}{2} \leqslant \cdots \leqslant 2 C+f_{r}^{0}=2 C+\mathbf{E} \int_{B_{R}}\left|D_{r, z} Y_{0}\right|^{2} v(\mathrm{~d} z)
$$

Thus,

$$
\sup _{n \in \mathbb{N} ; r \in[0, T]} \mathbf{E}\left[\int_{B_{R}}\left[\sup _{t \in[r, T]}\left|D_{r, z} Y_{t}^{n}\right|^{2}\right] v(\mathrm{~d} z)\right]<\infty .
$$

which implies (4.7) for sufficiently small $T$. For arbitrary $T>T_{0}$, by the similar argument above, we can see that

$$
\begin{aligned}
& \sup _{n \in \mathbb{N} ; r \in[0, T]} \mathbf{E}\left[\int_{B_{R}}\left[\sup _{t \in[r, T]}\left|D_{r, z} Y_{t}^{n}\right|^{2}\right] v(\mathrm{~d} z)\right] \leqslant \sup _{n \in \mathbb{N} ; r \in[0, T]} f_{r}^{n} \\
& \leqslant 2 C+\sup _{n \in \mathbb{N} ; r \in[0, T]} \mathbf{E} \int_{B_{R}}\left|D_{r, z} Y_{T-T_{0}}^{n}\right|^{2} v(\mathrm{~d} z) \\
& \leqslant \cdots \leqslant 2 C\left(\left[T / T_{0}\right]+1\right)+\sup _{r \in[0, T]} \mathbf{E} \int_{B_{R}}\left|D_{r, z} Y_{0}\right|^{2} v(\mathrm{~d} z)<\infty .
\end{aligned}
$$

So we complete our proof.

(ii) Choosing $\lambda$ sufficiently large, by $\left(\mathrm{A}_{4}\right)$ and (4.5), for any $z \in B_{r_{0}}$, we have

$$
\begin{aligned}
\left|\nabla_{y} g(y, z)\right| & \leqslant\|\nabla \sigma\|_{\infty}|z|+c(\lambda, \mu)(1-c(\lambda, \mu))^{-1}\left(\|\sigma\|_{\infty}^{\mu}|z|^{\mu}+\|\nabla \sigma\|_{\infty}|z|\right) \\
& \leqslant r_{0}\|\nabla \sigma\|_{\infty}^{-1}+C \cdot c(\lambda, \mu)<1 .
\end{aligned}
$$

This implies that for each $z \in \operatorname{supp} v \subseteq B_{r_{0}}$ the map $y \mapsto y+g(y, z)$ is homeomorphic and $\mathbb{I}+\nabla_{y} g(y, z)$ is invertible. Again by (4.5), for any $z \in B_{r_{0}},\left\|\nabla_{y} g(\cdot, z)\right\|_{\infty} \leqslant K(z) \asymp|z|^{\mu}$. Since $2 \mu>\alpha$, by (3.9),

$$
\int_{B_{r_{0}}} K(z)^{2} v(\mathrm{~d} z) \leqslant C \int_{B_{r_{0}}}|z|^{2 \mu} v_{2}(\mathrm{~d} z) \leqslant C r_{0}^{2 \mu-\alpha}<\infty .
$$


Noting that $\sigma \in C_{b}^{1+\delta}$, by (4.3) and the regularity estimates for $u$, one can also check that

$$
\left|\nabla_{y} g(y, z)-\nabla_{y} g\left(y^{\prime}, z\right)\right| \leqslant L(z)\left|y-y^{\prime}\right|^{\delta \wedge \mu}
$$

and $L(z) \asymp|z|^{\mu}$. So we also have

$$
\int_{B_{r_{0}}} L(z)^{2} v(\mathrm{~d} z)<\infty
$$

Thanks to [Kun04, Theorem 3.11], $\left\{Y_{t}(x)\right\}$ defines a $C^{1}$-stochastic flow. So $\left\{X_{t}(x)\right\}$ also forms a $C^{1}$-stochastic flow.

In [Dav07], Davie proved a remarkable result, which says that given a bounded Borel measurable mapping $b$, for almost all Brownian paths $W_{t}(\omega)$, there is a unique $\theta_{t}(\omega)$ satisfying the random ODE: $\mathrm{d} \theta_{t}(\omega)=b\left(\theta_{t}(\omega)+W_{t}(\omega)\right) \mathrm{d} t$. This type of uniqueness for SDEs with jumps was first studied by Priola in [Pri18]. In the light of [Pri18, Theorem 1.1], we show a path-by-path uniqueness result for (1.1) below.

Proposition 4.1. Suppose $Z$ is a non-degenerate symmetric $\alpha$-stable process, $\sigma=\mathbb{I}$ and $b \in C_{b}^{\beta}$ with $\beta \in\left(1-\frac{\alpha}{2}, 1\right)$. Then there is a $\mathbf{P}$-null set $\mathscr{N} \subseteq \Omega$ such that for any $\omega \notin \mathscr{N}$, the following $O D E$ :

$$
\frac{\mathrm{d} z_{t}(\omega)}{\mathrm{d} t}=b\left(z_{t}(\omega)+Z_{t}(\omega)\right), \quad z_{0}=x
$$

admits a unique solution in $C\left(\mathbb{R}_{+} ; \mathbb{R}^{d}\right)$.

Proof. Fix $\gamma \in\left(1-\frac{\alpha}{2}, \beta\right)$. By Remark 1.2 (2) and interpolation theorem, there is a constant $\lambda>$ such that the following resolvent equation

$$
\lambda u_{\lambda}-L u_{\lambda}-b \cdot \nabla u_{\lambda}=b
$$

has a solution $u_{\lambda} \in C_{b}^{\alpha+\gamma}$ satisfying $\left\|\nabla u_{\lambda}\right\|_{\infty}<1 / 3$. Thanks to [Pri18, Theorem 6.6 and Theorem 1.1], (4.8) has exactly one solution $z$ in $C\left(\mathbb{R}_{+} ; \mathbb{R}^{d}\right)$.

\section{ACKNOWLEDGEMENTS}

The author is very grateful to Professor Enrico Priola who read the first draft and pointed out some mistakes and also to Professor Moritz Kassmann and Professor Xicheng Zhang for their valuable discussion.

\section{REFERENCES}

[BCD11] Hajer Bahouri, Jean-Yves Chemin, and Raphaël Danchin. Fourier analysis and nonlinear partial differential equations, volume 343. Springer Science \& Business Media, 2011.

[BK05] Richard F Bass and Moritz Kassmann. Hölder continuity of harmonic functions with respect to operators of variable order. Communications in Partial Differential Equations, 30(8):1249-1259, 2005.

[BL02a] Richard Bass and David Levin. Transition probabilities for symmetric jump processes. Transactions of the American Mathematical Society, 354(7):2933-2953, 2002.

[BL02b] Richard F Bass and David A Levin. Harnack inequalities for jump processes. Potential Analysis, 17(4):375-388, 2002.

[BSW13] Björn Böttcher, René Schilling, and Jian Wang. Lévy matters. III. Lévy-Type Processes: Construction, Approximation and Sample Path Properties, volume 2099. Springer, 2013. 
[CCV11] Luis Caffarelli, Chi Hin Chan, and Alexis Vasseur. Regularity theory for parabolic nonlinear integral operators. Journal of the American Mathematical Society, 24(3):849-869, 2011.

[CK03] Zhen-Qing Chen and Takashi Kumagai. Heat kernel estimates for stable-like processes on d-sets. Stochastic Processes and their applications, 108(1):27-62, 2003.

[CMZ07] Qionglei Chen, Changxing Miao, and Zhifei Zhang. A new bernstein's inequality and the 2d dissipative quasi-geostrophic equation. Communications in mathematical physics, 271(3):821-838, 2007.

[CSZ18] Zhen-Qing Chen, Renming Song, and Xicheng Zhang. Stochastic flows for Lévy processes with Hölder drifts. Revista Matemática Iberoamericana, 34(4), 2018.

[CZZ21] Zhen-Qing Chen, Xicheng Zhang, and Guohuan Zhao. Supercritical SDEs driven by multiplicative stablelike Lévy processes. To apear in Transaction of American Mathematical Society., 2021.

[Dav07] Alexander M Davie. Uniqueness of solutions of stochastic differential equations. International Mathematics Research Notices, 2007, 2007.

[DJZ18] Hongjie Dong, Tianling Jin, and Hong Zhang. Dini and schauder estimates for nonlocal fully nonlinear parabolic equations with drifts. Analysis \& PDE, 11(6):1487-1534, 2018.

[DK13] Hongjie Dong and Doyoon Kim. Schauder estimates for a class of non-local elliptic equations. Discrete Contin. Dyn. Syst., 33(6):2319-2347, 2013.

[DNØP09] Giulia Di Nunno, Bernt Karsten Øksendal, and Frank Proske. Malliavin calculus for Lévy processes with applications to finance, volume 2. Springer, 2009.

[dRMP20] Paul-Éric Chaudru de Raynal, Stephane Menozzi, and Enrico Priola. Schauder estimates for drifted fractional operators in the supercritical case. Journal of Functional Analysis, 278(8):108425, 2020.

[HL18] Xing Huang and Zhong-Wei Liao. The Euler-Maruyama method for SDEs with Hölder drift and $\alpha$-stable noise. Stochastic Analysis and Applications, 36(1):28-39, 2018.

[HP14] Sven Haadem and Frank Proske. On the construction and Malliavin differentiability of solutions of Lévy noise driven SDEs with singular coefficients. Journal of Functional Analysis, 266(8):5321-5359, 2014.

[HWW20] Zimo Hao, Zhen Wang, and Mingyan Wu. Schauder's estimates for nonlocal equations with singular Lévy measures. arXiv preprint arXiv:2002.09887, 2020.

[Jac05] Niels Jacob. Pseudo differential operators and Markov processes, volume I-III. Imperial College Press, 2001-2005.

[JS01] Niels Jacob and René L Schilling. Lévy-type processes and pseudodifferential operators. In Lévy processes: Theory and Applications, pages 139-168. Springer, 2001.

[Kas09] Moritz Kassmann. A priori estimates for integro-differential operators with measurable kernels. Calculus of Variations and Partial Differential Equations, 34(1):1-21, 2009.

[KR05] Nicolai V Krylov and Michael Röckner. Strong solutions of stochastic equations with singular time dependent drift. Probability Theory and Related Fields, 131(2):154-196, 2005.

[KS19] Franziska Kühn and René L Schilling. Strong convergence of the Euler-Maruyama approximation for a class of Lévy-driven SDEs. Stochastic Processes and their Applications, 129(8):2654-2680, 2019.

[Kul19] Alexei M Kulik. On weak uniqueness and distributional properties of a solution to an SDE with $\alpha$-stable noise. Stochastic Processes and their Applications, 129(2):473-506, 2019.

[Kun04] Hiroshi Kunita. Stochastic differential equations based on Lévy processes and stochastic flows of diffeomorphisms. In Real and stochastic analysis, pages 305-373. Springer, 2004.

[LZ19] Chengcheng Lin and Guohuan Zhao. Nonlocal elliptic equation in Hölder space and the martingale problem. arXiv preprint arXiv:1907.00588, 2019.

[MBP10] Thilo Meyer-Brandis and Frank Proske. Construction of strong solutions of SDE's via Malliavin calculus. Journal of Functional Analysis, 258(11):3922-3953, 2010.

[MNP15] Salah-Eldin A Mohammed, Torstein K Nilssen, and Frank N Proske. Sobolev differentiable stochastic flows for SDEs with singular coefficients: Applications to the transport equation. The Annals of Probability, 43(3):1535-1576, 2015. 
[MP92] Remigijus Mikulevicius and Henrikas Pragarauskas. On the Cauchy problem for certain integrodifferential operators in Sobolev and Hölder spaces. Lithuanian Mathematical Journal, 32(2):238-264, 1992.

$\left[\mathrm{MPMBN}^{+}\right.$13] Olivier Menoukeu-Pamen, Thilo Meyer-Brandis, Torstein Nilssen, Frank Proske, and Tusheng Zhang. A variational approach to the construction and Malliavin differentiability of strong solutions of SDE's. Mathematische Annalen, 357(2):761-799, 2013.

[MX18] R Mikulevicius and Fanhui Xu. On the rate of convergence of strong Euler approximation for SDEs driven by Lévy processes. Stochastics, 90(4):569-604, 2018.

[Pri12] Enrico Priola. Pathwise uniqueness for singular SDEs driven by stable processes. Osaka Journal of Mathematics, 49(2):421-447, 2012.

[Pri15] Enrico Priola. Stochastic flow for SDEs with jumps and irregular drift term. Banach Center Publications, 105(1):421-44193-210, 2015.

[Pri18] Enrico Priola. Davie's type uniqueness for a class of SDEs with jumps. Annales de l'Institut Henri Poincaré, Probabilités et Statistiques, 54(2):694-725, 2018.

[Sil06] Luis Silvestre. Hölder estimates for solutions of integro-differential equations like the fractional laplace. Indiana University mathematics journal, pages 1155-1174, 2006.

[Sil12] Luis Silvestre. On the differentiability of the solution to an equation with drift and fractional diffusion. Indiana University Mathematics Journal, 61(2):557-584, 2012.

[SS10] René L Schilling and Alexander Schnurr. The symbol associated with the solution of a stochastic differential equation. Electronic Journal of Probability, 15:1369-1393, 2010.

[Tri92] Hans. Triebel. Theory of function spaces II, volume 84. Birkhäuser Basel, 1992.

[TTW74] Hiroshi Tanaka, Masaaki Tsuchiya, and Shinzo Watanabe. Perturbation of drift-type for Lévy processes. Journal of Mathematics of Kyoto University, 14(1):73-92, 1974.

[Ver80] Alexander Yur'evich Veretennikov. On strong solutions and explicit formulas for solutions of stochastic integral equations. Matematicheskii Sbornik, 153(3):434-452, 1980.

[XXZZ20] Pengcheng Xia, Longjie Xie, Xicheng Zhang, and Guohuan Zhao. $L^{q}\left(L^{p}\right)$-theory of stochastic differential equations. Stochastic Processes and their Applications, 130(8):5188-5211, 2020.

[Zha11] Xicheng Zhang. Stochastic homeomorphism flows of SDEs with singular drifts and Sobolev diffusion coefficients. Electronic Journal of Probability, 16:1096-1116, 2011.

[Zha13] Xicheng Zhang. Stochastic differential equations with Sobolev drifts and driven by $\alpha$-stable processes. Annales de l'IHP Probabilités et statistiques, 49(4):1057-1079, 2013.

[Zha16] Xicheng Zhang. Stochastic differential equations with Sobolev diffusion and singular drift and applications. The Annals of Applied Probability, 26(5):2697-2732, 2016.

[Zha19] Guohuan Zhao. Weak uniqueness for SDEs driven by supercritical stable processes with Hölder drifts. Proceedings of the American Mathematical Society, 147(2):849-860, 2019.

[Zvo74] Alexander K Zvonkin. A transformation of the phase space of a diffusion process that removes the drift. Mathematics of the USSR-Sbornik, 22(1):129, 1974.

[ZZ18] Xicheng Zhang and Guohuan Zhao. Dirichlet problem for supercritical nonlocal operators. arXiv preprint arXiv:1809.05712, 2018.

[ZZ21] Xicheng Zhang and Guohuan Zhao. Stochastic lagrangian path for leray's solutions of 3d navier-stokes equations. Communications in Mathematical Physics, 381(2):491-525, 2021.

Guohuan Zhao: Department of Mathematics, Bielefeld University, Germany, Email: ZhaoguoHUAN@GMAIL.COM 